\title{
Correlation Inequalities and Applications to Vector-Valued Gaussian Random Variables and Fractional Brownian Motion
}

\author{
Mark Veraar
}

Received: 30 January 2008 / Accepted: 10 February 2009 / Published online: 5 March 2009

(C) The Author(s) 2009. This article is published with open access at Springerlink.com

\begin{abstract}
In this paper we extend certain correlation inequalities for vector-valued Gaussian random variables due to Kolmogorov and Rozanov. The inequalities are applied to sequences of Gaussian random variables and Gaussian processes. For sequences of Gaussian random variables satisfying a correlation assumption, we prove a Borel-Cantelli lemma, maximal inequalities and several laws of large numbers. This extends results of Beśka and Ciesielski and of Hytönen and the author. In the second part of the paper we consider a certain class of vector-valued Gaussian processes which are $\alpha$-Hölder continuous in $p$-th moment. For these processes we obtain Besov regularity of the paths of order $\alpha$. We also obtain estimates for the moments in the Besov norm. In particular, the results are applied to vectorvalued fractional Brownian motions. These results extend earlier work of Ciesielski, Kerkyacharian and Roynette and of Hytönen and the author.
\end{abstract}

Keywords Correlation inequalities • Gebelein's inequality • Gaussian random variables • Maximal inequalities $\cdot$ Law of large numbers • Type and cotype $\cdot$ Gaussian processes $\cdot$ Fractional Brownian motion • Besov-Orlicz spaces $\cdot$ Sample path $\cdot$ Non-separable Banach space

Mathematics Subject Classifications (2000) Primary: 60G15 • Secondary: 46E35 • $60 \mathrm{~B} 12 \cdot 60 \mathrm{G} 17$

This work originated during the author's stay at the IMPAN institute in Warsaw which was supported by the Research Training Network MRTN-CT-2004-511953. Part of the work has been carried out during the author's stay at the University of Karlsruhe as an Alexander von Humboldt fellow.

M. Veraar $(\bowtie)$

Delft Institute of Applied Mathematics,

Delft University of Technology,

P.O. Box 5031, 2600 GA Delft, The Netherlands

e-mail: M.C.Veraar@tudelft.nl,mark@profsonline.nl 


\section{Introduction}

Let $(\Omega, \mathcal{F}, \mathbb{P})$ be a probability space. Let $(\xi, \eta)$ be a centered Gaussian vector in $\mathbb{R}^{2}$ with $\mathbb{E} \xi^{2}=\mathbb{E} \eta^{2}=1$. Let the correlation number between $\xi$ and $\eta$ be defined as $\rho=|\mathbb{E} \xi \eta|$. The Gebelein inequality [13] (also see [2]) states that

$$
|\mathbb{E} f(\xi) g(\eta)| \leq \rho\left(\mathbb{E}|f(\xi)|^{2}\right)^{\frac{1}{2}}\left(\mathbb{E}|g(\eta)|^{2}\right)^{\frac{1}{2}}
$$

for all $f, g: \mathbb{R} \rightarrow \mathbb{R}$ such that $f(\xi), g(\eta) \in L^{2}(\Omega)$ and $\mathbb{E} f(\xi)=g(\eta)=0$. In other words, if Eq. 1.1 holds for all linear $f$ and $g$, then it holds for all centered $f$ and $g$ which are in $L^{2}(\mu)$, where $\mu$ is the standard Gaussian measure on $\mathbb{R}$. In the latter formulation, the inequality (1.1) is extended by Kolmogorov and Rozanov [16] to the multidimensional Gaussian setting (also see [9]). We will extend the result also to vector-valued $f$ and $g$. The Gebelein inequality gives a method to obtain control over the rate of dependence of $\xi$ and $\eta$. In particular, this is illustrated by the following consequence due to Kolmogorov and Rozanov:

$$
\mathbb{P}(\xi \in A, \eta \in B) \leq \frac{\rho}{4} \mathbb{P}(\xi \in A) \mathbb{P}(\eta \in B), \quad A, B \in \mathcal{B}_{\mathbb{R}} .
$$

In $[1,2]$, Beśka and Ciesielski have studied sequences of real valued centered Gaussian random variables $\left(\xi_{i}\right)_{n \geq 1}$ with $\mathbb{E} \xi_{i}^{2}=1$ and $\left(\xi_{1}, \xi_{2}, \ldots, \xi_{n}\right)$ is Gaussian for all $n$. Let $\rho$ be the matrix defined as $\rho_{i j}=\left|\mathbb{E} \xi_{i} \xi_{j}\right|$. Under the assumption that

$$
C=\sup _{i \geq 1} \sum_{j \geq 1} \rho_{i j}<\infty
$$

the authors extend several result for independent random variables to the above setting. For example the Borel-Cantelli lemma and several laws of large number are proved for sequences of the form $\left(f_{n}\left(\xi_{n}\right)\right)_{n \geq 1}$, where $f_{n}: \mathbb{R} \rightarrow \mathbb{R}$ is such that $f_{n}\left(\xi_{n}\right) \in$ $L^{2}(\Omega)$.

In the first part of this paper, we will extend the above results to the setting where $\left(\xi_{n}\right)_{n \geq 1}$ are Gaussian random variables in a locally convex space $X$ and $f_{n}: X \rightarrow Y$, where $Y$ is a Banach space. However, in some cases we have to assume more structure of the space $Y$. We also extend a maximal inequality due to Hytönen and the author [15] to the above setting. The result is a two-sided inequality for $\mathbb{E} \sup _{n \geq 1}\left\|\xi_{n}\right\|$ in terms of the moments and weak variance of $\xi_{n}$.

In [5] Ciesielski proved that the standard Brownian motion $W$ on $[0,1]$ has paths in the Besov space $B_{p, \infty}^{\frac{1}{2}}(0,1)$ for all $p<\infty$. Later Roynette [27] characterized all triples $(\alpha, p, q)$ for which $W \in B_{p, q}^{\alpha}(0,1)$. In [6] Ciesielski improved his result by proving a Besov-Orlicz regularity result for $W$. The proofs of the above results depend on non-trivial norm equivalences for Besov norms. In [15] Hytönen and the author found a new method which gives a way to obtain the above Besov regularity results directly from the definition of the Besov space. Also vector-valued Brownian motions are considered there. Moreover, maximal inequalities are applied to obtain two-sided estimates for the first moment of $W$ in the Besov norm $B_{p, \infty}^{\frac{1}{2}}$ and a certain Besov-Orlicz space.

As a second application of Eq. 1.1 one can study regularity of Gaussian processes.

For Gaussian processes which do not have independent increments one can use Eq. 1.1 to obtain Besov regularity results. This has been done for a certain class of 
Gaussian processes by Ciesielski, Kerkyacharian and Roynette in [7]. In particular, they prove that the fractional Brownian motion with Hurst parameter $H \in(0,1)$ has paths in $B_{p, \infty}^{H}(0,1)$. Again the proofs depend on the norm equivalences for Besov spaces.

In the second part of this paper we will consider a certain class of Gaussian process $G:[0,1] \times \Omega \rightarrow X$, where $X$ is a Banach space. Assuming that $G$ is $\alpha$-Hölder continuous in $p$-th moment, we will show that $G$ has paths in $B_{p, \infty}^{\alpha}(0,1 ; X)$ and in a certain Besov-Orlicz space. We will use the methods from [15] combined with the vector-valued generalization of Eq. 1.1 to obtain the Besov regularity results directly from the definition of the Besov space. We will also obtain two-sided estimates for the first moment of $G$ in the Besov and Besov-Orlicz norm. In particular, we apply the results to vector-valued fractional Brownian motions.

The paper is organized in the following way. Some preliminaries are discussed in Section 2. In Section 3 we prove a generalization of Eq. 1.1 to the vector-valued situation. In Section 4 we consider sequences of Gaussian random variables with values in a locally convex space under the assumption that Eq. 1.2 holds. In the last and main Section 5, we consider the Besov regularity of Gaussian processes.

For convenience we will only consider real vector spaces in this paper.

\section{Preliminaries}

We will write $a \lesssim b$ if there exists a universal constant $C>0$ such that $a \leq C b$, and $a \approx b$ if $a \lesssim b \lesssim a$. If the constant $C$ is allowed to depend on some parameter $t$, we write $a \lesssim_{t} b$ and $a{\sim_{t}}_{b}$ instead.

For sake of completeness, we recall the definitions of Orlicz and Besov spaces that we will need. We use the same notation and definitions as in [15].

\subsection{Orlicz Spaces}

We briefly recall the definition of Orlicz spaces. More details can be found in $[18,24,29]$.

Let $(S, \Sigma, \mu)$ be a $\sigma$-finite measure space and let $X$ be a Banach space. Let $\Phi$ : $\mathbb{R} \rightarrow \mathbb{R}_{+}$be an even convex function such that $\Phi(0)=0$ and $\lim _{x \rightarrow \infty} \Phi(x)=\infty$. The Orlicz space $L^{\Phi}(S ; X)$ is defined as the set of all strongly measurable functions $f$ : $S \rightarrow X$ (identifying functions which are equal $\mu$-a.e.) with the property that there exists a $\delta>0$ such that

$$
M_{\Phi}(f / \delta):=\int_{S} \Phi(\|f(s)\| / \delta) d \mu(s)<\infty .
$$

This space is a vector space and we define

$$
\rho_{\Phi}(f)=\inf \left\{\delta>0: M_{\Phi}(f / \delta) \leq 1\right\} .
$$

The mapping $\rho_{\Phi}$ defines a norm on $L^{\Phi}(S ; X)$ and it turns $L^{\Phi}(S ; X)$ into a Banach space. It is usually referred to as the Luxemburg norm.

For $f \in L^{\Phi}(S ; X)$ we also define the Orlicz norm

$$
\|f\|_{\Phi}=\inf _{\delta>0}\left\{\frac{1}{\delta}\left(1+M_{\Phi}(\delta f)\right)\right\} .
$$


Usually the Orlicz norm is defined in a different way using duality, but the above norm gives exactly the same number (cf. [24, Theorem III.13]). The two norms are equivalent (cf. [15, Lemma 2.1]).

\subsection{Besov Spaces}

We recall the definition of the vector-valued Besov spaces. For the real case we refer to [28] and for the vector-valued Besov space we will give the treatise from [17].

Let $X$ be a Banach space and let $I=(0,1)$. For $\alpha \in(0,1), p, q \in[1, \infty]$ the vectorvalued Besov space $B_{p, q}^{\alpha}(I ; X)$ is defined as the space of all functions $f \in L^{p}(I ; X)$ for which the seminorm (with the usual modification for $q=\infty$ )

$$
\left(\int_{0}^{1}\left(t^{-\alpha} \omega_{p}(f, t)\right)^{q} \frac{d t}{t}\right)^{1 / q}
$$

is finite. Here

$$
\omega_{p}(f, t)=\sup _{|h| \leq t}\|f(\cdot+h)-f(\cdot)\|_{L^{p}(I(h) ; X)}
$$

with $I(h)=\{s \in I: s+h \in I\}$. The sum of the $L^{p}$-norm and this seminorm turn $B_{p, q}^{\alpha}(I ; X)$ into a Banach space. By a dyadic approximation argument (see [17, Corollary 3.b.9]) one can show that the above seminorm is equivalent to

$$
\|f\|_{p, q, \alpha}:=\left(\sum_{n \geq 0}\left(2^{n \alpha}\left\|f\left(\cdot+2^{-n}\right)-f(\cdot)\right\|_{L^{p}\left(I\left(2^{-n}\right) ; X\right)}\right)^{q}\right)^{1 / q} .
$$

For the purposes below it will be convenient to take

$$
\|f\|_{B_{p, q}^{\alpha}(I ; X)}=\|f\|_{L^{p}(I ; X)}+\|f\|_{p, q, \alpha}
$$

as a Banach space norm on $B_{p, q}^{\alpha}(I ; X)$.

For $0<\beta<\infty$, we also introduce the exponential Orlicz and Orlicz-Besov (semi)norms

$$
\begin{aligned}
\|f\|_{\mathfrak{L}^{\Phi_{\beta}(I ; X)}} & :=\sup _{p \geq 1} p^{-1 / \beta}\|f\|_{L^{p}(I ; X)}, \\
\|f\|_{\Phi_{\beta}, \infty, \alpha} & :=\sup _{n \geq 1} 2^{\alpha n}\left\|f-f\left(\cdot-2^{-n}\right)\right\|_{\mathfrak{L}^{\Phi_{\beta}\left(I\left(2^{-n}\right) ; X\right)}}=\sup _{p \geq 1} p^{-1 / \beta}\|f\|_{p, \infty, \alpha},
\end{aligned}
$$

and finally the Orlicz-Besov norm

$$
\|f\|_{B_{\Phi_{\beta}, \infty}^{\alpha}(I ; X)}:=\sup _{p \geq 1} p^{-1 / \beta}\|f\|_{B_{p, \infty}^{\alpha}(I ; X)} \approx\|f\|_{\mathfrak{L}^{\Phi_{\beta}(I ; X)}}+\|f\|_{\Phi_{\beta}, \infty, \alpha} .
$$

Because of the inequalities between different $L^{p}$ norms, it is immediate that we have equivalent norms above, whether we understand $p \geq 1$ as $p \in[1, \infty)$ or $p \in\{1,2, \ldots\}$. For definiteness and later convenience, we choose the latter.

The above-given norm of $\mathfrak{L}^{\Phi_{\beta}}(I ; X)$ is equivalent to the usual norm of the Orlicz space $L^{\Phi_{\beta}}(I ; X)$ from Subsection 2.1 where $\Phi_{\beta}(x)=\exp \left(|x|^{\beta}\right)-1$ for $\beta \geq 1$. 


\section{Correlation Inequalities}

Let $X$ and $Y$ be locally convex spaces. Let $(\Omega, \mathcal{F}, \mathbb{P})$ be a probability space. A measurable mapping $\xi: \Omega \rightarrow X$ is said to be a centered Gaussian random variable if for all $x^{*} \in X^{*},\left\langle\xi, x^{*}\right\rangle$ is a centered Gaussian random variable. A probability measure $\mu$ on $\mathcal{B}(X)$ is called a centered Gaussian measure, if for all $x^{*} \in X^{*}$ its image $\mu \circ\left(x^{*}\right)^{-1}$ is a centered Gaussian measure on $\mathcal{B}(\mathbb{R})$. In this section we will usually assume that $\xi$ and $\mu$ are also Radon.

An extensive theory for Gaussian measures on locally convex spaces can be found in [3].

Let $\xi: \Omega \rightarrow X$ and $\eta: \Omega \rightarrow Y$ be such that $(\xi, \eta)$ is a centered Gaussian random variable in $X \times Y$. Define the (absolute) correlation between $\xi$ and $\eta$ as

$$
\rho=\sup _{x^{*} \in X^{*}, y^{*} \in Y^{*}} \frac{\left|\mathbb{E}\left\langle\xi, x^{*}\right\rangle\left\langle\eta, y^{*}\right\rangle\right|}{\left(\mathbb{E}\left|\left\langle\xi, x^{*}\right\rangle\right|^{2}\right)^{\frac{1}{2}}\left(\mathbb{E}\left|\left\langle\eta, y^{*}\right\rangle\right|^{2}\right)^{\frac{1}{2}}},
$$

with the convention $\frac{0}{0}=0$. Equivalently one can write

$\rho=\inf \left\{r \geq 0:\left|\mathbb{E}\left\langle\xi, x^{*}\right\rangle\left\langle\eta, y^{*}\right\rangle\right| \leq r\left(\mathbb{E}\left|\left\langle\xi, x^{*}\right\rangle\right|^{2}\right)^{\frac{1}{2}}\left(\mathbb{E}\left|\left\langle\eta, y^{*}\right\rangle\right|^{2}\right)^{\frac{1}{2}}, x^{*} \in X^{*}, y^{*} \in Y^{*}\right\}$.

Clearly, $\rho \in[0,1]$ and $\rho=0$ if and only if $\xi$ and $\eta$ are independent. If we want to emphasize the dependence of $\rho$ on $\xi$ and $\eta$ we write $\rho(\xi, \eta)$ instead of $\rho$.

If $X=\mathbb{R}^{m}$ and $Y=\mathbb{R}^{n}$ and if $\xi$ and $\eta$ are non-degenerate, then one can check that $\rho=\left\|A_{11}^{-\frac{1}{2}} A_{12} A_{22}^{-\frac{1}{2}}\right\|$ with $A_{11}=\mathbb{E} \xi \xi^{t}, A_{12}=\mathbb{E} \xi \eta^{t}$ and $A_{22}=\mathbb{E} \eta \eta^{t}$.

In the next result we will extend [6, Theorem 4.6] to the vector valued situation. It will play a key role in the proof of Theorem 3.4. We will need the setting of UMD Banach spaces. We refer to [4] for details on UMD spaces. UMD stands for unconditional martingale differences. Example of UMD space are Hilbert spaces and reflexive $L^{p}$-spaces. If $Z$ is a UMD space, then its dual $Z^{*}$ is also UMD.

Proposition 3.1 (Gebelein inequality, preliminary version) Let $\mu$ be a centered Gaussian Radon measure on a locally convex space $X$. Let $Z$ be a UMD Banach space and let $p, p^{\prime} \in(1, \infty)$ be such that $\frac{1}{p}+\frac{1}{p^{\prime}}=1$. Let $(\xi, \eta)$ be a Gaussian random variable such that $\xi$ and $\eta$ have distribution $\mu$. Assume that there is a number $r \in[-1,1]$ such that for all $x^{*} \in X^{*}$ and $y^{*} \in X^{*}$

$$
\mathbb{E}\left\langle\xi, x^{*}\right\rangle\left\langle\eta, y^{*}\right\rangle=r \mathbb{E}\left\langle\xi, x^{*}\right\rangle\left\langle\xi, y^{*}\right\rangle .
$$

Then for all $f \in L^{p}(\mu ; Z)$ and $g \in L^{p^{\prime}}\left(\mu ; Z^{*}\right)$ such that $\mathbb{E} f(\xi)=0$ and $\mathbb{E} g(\eta)=0$ the following estimate holds

$$
|\mathbb{E}\langle f(\xi), g(\eta)\rangle| \leq C|r|\|f(\xi)\|_{L^{p}(\Omega ; Z)}\|g(\eta)\|_{L^{p^{\prime}}\left(\Omega ; Z^{*}\right)},
$$

where $C$ depends on $p$ and $Z$ only. Moreover, if $f$ or $g$ is even, then

$$
|\mathbb{E}\langle f(\xi), g(\eta)\rangle| \leq C^{2}|r|^{2}\|f(\xi)\|_{L^{p}(\Omega ; Z)}\|g(\eta)\|_{L^{p^{\prime}}\left(\Omega ; Z^{*}\right)} .
$$

Notice that from Eqs. 3.1 and 3.2 it follows that $\rho=|r|$.

The following will be clear from the proof of Proposition 3.1. 
Remark 3.2 Instead of the function $\langle\cdot, \cdot\rangle: Z \times Z^{*} \rightarrow \mathbb{R}$, one may consider an arbitrary bilinear mapping $\Gamma: Z_{1} \times Z_{2} \rightarrow Z_{3}$, where $Z_{1}, Z_{2}$ are UMD Banach spaces and $Z_{3}$ is a Banach space.

Remark 3.3 We do not know whether the assumption that $Z$ is a UMD space is necessary in Proposition 3.1. We do know that the result fails for $p=1$ in general. Indeed, in the case $X=Z=\mathbb{R}$ and $\mu$ is the standard Gaussian measure on $\mathbb{R}$, then as in the proof below the estimate (3.3) implies that

$$
\left\|Q^{\mu}(t) f\right\|_{L^{1}(\mu)} \leq C e^{-t}\|f\|_{L^{1}(\mu)}
$$

for all $f \in L^{1}(\mu)$ with mean zero. This inequality cannot hold, since the point spectrum of the generator of the Ornstein-Uhlenbeck operator is $\{\lambda \in \mathbb{C}: \operatorname{Re} \lambda<0\}$. (cf. [8, Theorem 3] and [22, Theorem 5.1]).

Proof We may assume $r \neq 0$. First assume $r>0$. Let $\tilde{\xi}$ and $\tilde{\eta}$ be independent copies of $\xi$. Then the Gaussian random variables $\left(r \tilde{\eta}+\sqrt{1-r^{2}} \tilde{\xi}, \tilde{\eta}\right)$ and $(\xi, \eta)$ have the same distribution and therefore

$$
\mathbb{E}\langle f(\xi), g(\eta)\rangle=\mathbb{E}\left\langle f\left(r \tilde{\eta}+\sqrt{1-r^{2}} \tilde{\xi}\right), g(\tilde{\eta})\right\rangle=\left\langle Q_{Z}^{\mu}(t) f, g\right\rangle_{\left(L^{p}(\mu ; Z), L^{p^{\prime}}\left(\mu ; Z^{*}\right)\right)} .
$$

Here $t=-\ln (r)$ and $\left(Q_{Z}^{\mu}(t)\right)_{t \geq 0}$ on $L^{p}(\mu ; Z)$ is the vector-valued extension of the the Ornstein-Uhlenbeck semigroup $\left(Q^{\mu}(t)\right)_{t \geq 0}$ on $L^{p}(\mu)$, i.e.

$$
Q^{\mu}(t) g(x)=\int_{X} g\left(e^{-t} x+\sqrt{1-e^{-2 t}} y\right) d \mu(y) .
$$

Note that by positivity of $Q_{\mu}(t), Q_{Z}^{\mu}(t)=Q_{\mu}(t) \otimes I$ extends to a contraction on $L^{p}(\mu ; Z)$. It follows from the Hölder's inequality that

$$
\left|\mathbb{E}\langle f(\xi), g(\eta)\rangle_{\left(L^{p}(\mu ; Z), L^{p^{\prime}\left(\mu ; Z^{*}\right)}\right)}\right| \leq\left\|Q_{Z}^{\mu}(t) f\right\|_{L^{p}(\mu ; Z)}\|g\|_{L^{p^{\prime}\left(\mu ; Z^{*}\right)}} .
$$

Now since $Z$ is a UMD space and $p \in(1, \infty)$, it follows from [21, Lemma 6.4] (also see $[23$, Lemma 1.4.1]) and $\mathbb{E} f(\xi)=0$ that

$$
\left\|Q_{Z}^{\mu}(t) f\right\|_{L^{p}(\mu ; Z)} \leq C e^{-t}\|f\|_{L^{p}(\mu ; Z)} .
$$

This implies Eq. 3.3. To obtain Eq. 3.4 we first assume that $f$ is even. Then [21, Lemma 6.4] gives that

$$
\left\|Q_{Z}^{\mu}(t) f\right\|_{L^{p}(\mu ; Z)} \leq C^{2} e^{-2 t}\|f\|_{L^{p}(\mu ; Z)}
$$

If $g$ is even, we have to argue as above but replacing the roles of $f$ and $g$.

If $r<0$, then one can reduce to the case $r>0$ if one replaces $\xi$ and $f(x)$ by $-\xi$ and $f(-x)$ respectively.

In the next result we extend the Gebelein inequality to the general setting. In the case $Z=\mathbb{R}$, this result is due to Kolmogorov and Rozanov [16] (see also [9, p. 66]) where it is formulated in a more geometric language. Also in the case $Z=\mathbb{R}$, our proof is of interest since it differs from the proof in $[9,16]$. 
Theorem 3.4 (Gebelein inequality, general version) Let $\xi$ and $\eta$ be centered Gaussian Radon random variables with values in locally convex spaces $X$ and $Y$ respectively, and such that $(\xi, \eta)$ has a Gaussian distribution as well. Let $Z$ be a UMD space and let $p \in(1, \infty)$. If $f: X \rightarrow Z$ and $g: Y \rightarrow Z^{*}$ are such that $f(\xi) \in L^{p}(\omega ; Z)$ and $g(\eta) \in L^{2}\left(\Omega ; Z^{*}\right)$ and $\mathbb{E} f(\xi)=\mathbb{E} g(\eta)=0$, then

$$
|\mathbb{E}\langle f(\xi), g(\eta)\rangle| \leq C \rho\|f(\xi)\|_{L^{p}(\Omega ; Z)}\|g(\eta)\|_{L^{p^{\prime}}\left(\Omega ; Z^{*}\right)},
$$

where $\rho$ is as in Eq. 3.1 and $C$ only depends on $p$ and $Z$. Moreover, if $f$ or $g$ is even, then

$$
|\mathbb{E}\langle f(\xi), g(\eta)\rangle| \leq C^{2} \rho^{2}\|f(\xi)\|_{L^{p}(\Omega ; Z)}\|g(\eta)\|_{\left.L^{p^{\prime}\left(\Omega ; Z^{*}\right.}\right)}
$$

It will be clear from the proof that the same Remark 3.2 applies to Theorem 3.4.

Proof By approximation we may reduce to the case where $f$ and $g$ can be written as

$$
f(x)=\phi\left(\left\langle x, x_{1}^{*}\right\rangle, \ldots,\left\langle x, x_{m}^{*}\right\rangle\right), g(y)=\psi\left(\left\langle y, y_{1}^{*}\right\rangle, \ldots,\left\langle y, y_{n}^{*}\right\rangle\right)
$$

where $\phi: \mathbb{R}^{m} \rightarrow Z$ and $\psi: \mathbb{R}^{n} \rightarrow Z^{*}$ are measurable and $x_{1}^{*}, \ldots, x_{m}^{*} \in X^{*}$ and $y_{1}^{*}, \ldots, y_{n}^{*} \in Y^{*}$ are such that $\left(\left\langle\xi, x_{1}^{*}\right\rangle, \ldots,\left\langle\xi, x_{m}^{*}\right\rangle\right)$ and $\left(\left\langle\eta, y_{1}^{*}\right\rangle, \ldots,\left\langle\xi, y_{n}^{*}\right\rangle\right)$ are standard Gaussian random variables in $\mathbb{R}^{m}$ and $\mathbb{R}^{n}$ respectively.

By the above observation it suffices to consider the case where $X=\mathbb{R}^{m}$ and $Y=\mathbb{R}^{n}$ and $\xi$ and $\eta$ are standard Gaussian random variables, where possibly $\rho$ in the approximated case is smaller than the original $\rho$. We may assume $\rho>0$. Let

$$
A=\mathbb{E}(\xi, \eta)\left(\xi^{t} \eta^{t}\right)=\left(\begin{array}{cc}
I_{m} & A_{12} \\
A_{12}^{t} & I_{n}
\end{array}\right)
$$

be the covariance block matrix of the Gaussian random variable $(\xi, \eta)$, i.e. $I_{m}=$ $\mathbb{E} \xi \xi^{t}, A_{12}=\mathbb{E} \xi \eta^{t}$ and $I_{n}=\mathbb{E} \eta \eta^{t}$. One easily checks that $\rho=\left\|A_{12}\right\|$. Let $\xi^{1}, \xi^{2}, \xi^{3}, \xi^{4}$ independent standard Gaussian vectors, where $\xi^{1}, \xi^{3}$ take values in $\mathbb{R}^{m}$ and $\xi^{2}, \xi^{4}$ take values in $\mathbb{R}^{n}$. Let $\tilde{\xi}=\left(\xi^{1}, \xi^{2}\right)$ and $\tilde{\eta}=\rho \tilde{\xi}+\sqrt{1-\rho^{2}}\left(\xi^{3}, \xi^{4}\right)$. We claim that there exists an $n \times(m+n)$ matrix $B=\left(B_{11} B_{12}\right)$ such that $\left(\xi^{1}, B \tilde{\eta}\right) \stackrel{D}{=}(\xi, \eta)$. From this the result would follow. Indeed, let $\tilde{f}: \mathbb{R}^{m} \times \mathbb{R}^{n} \rightarrow Z$ and $\tilde{g}: \mathbb{R}^{m} \times \mathbb{R}^{n} \rightarrow Z^{*}$ be defined as $\tilde{f}(x, y)=f(x)$ and $\tilde{g}(x, y)=g(B(x, y))$, it follows from Proposition 3.1 that

$$
\begin{aligned}
|\mathbb{E}\langle f(\xi), g(\eta)\rangle| & =|\mathbb{E}\langle\tilde{f}(\tilde{\xi}), \tilde{g}(\tilde{\eta})\rangle| \leq C \rho\|\tilde{f}(\tilde{\xi})\|_{L^{p}(\Omega ; Z)}\|\tilde{g}(\tilde{\eta})\|_{L^{p^{\prime}}\left(\Omega ; Z^{*}\right)} \\
& =C \rho\|f(\xi)\|_{L^{p}(\Omega ; Z)}\|g(\eta)\|_{L^{p^{\prime}}\left(\Omega ; Z^{*}\right)},
\end{aligned}
$$

which shows Eq. 3.6. The same argument combined with Eq. 3.4 gives Eq. 3.7.

For the claim the two Gaussian random variables $\left(\xi^{1}, B \tilde{\eta}\right)$ and $(\xi, \eta)$ have to have the same covariance structure. Therefore, $B$ has to satisfy

$$
A_{22}=\mathbb{E} \eta \eta^{t}=B \tilde{\eta} \tilde{\eta}^{t} B^{t}=B B^{t}=B_{11} B_{11}^{t}+B_{12} B_{12}^{t}
$$

and

$$
A_{12}=\mathbb{E} \xi \eta^{t}=\mathbb{E} \xi^{1}(B \tilde{\eta})^{t}=\mathbb{E} \xi^{1} \tilde{\eta}^{t} B^{t}=\rho\left(I_{m} 0\right) B^{t}=\rho B_{11}^{t} .
$$

One easily checks that $B_{11}=\rho^{-1} A_{12}^{t}$ and $B_{12}=P\left(I-\rho^{-2} E\right)^{\frac{1}{2}}$ satisfy these equations. Here $P$ is a orthogonal matrix and $E$ a diagonal matrix such that $A_{12}^{t} A_{12}=$ $P E P^{t}$. Since $\max _{i} E_{i i}=\left\|A_{12}\right\|^{2}=\rho^{2}, B_{12}$ is well-defined. 
For later reference it will be convenient to state the case that $Z$ is a Hilbert space and $p=2$ separately. In this case the result holds with constant $C=1$.

Corollary 3.5 Let $\xi$ and $\eta$ be centered Gaussian Radon random variables with values in locally convex spaces $X$ and $Y$ respectively, and such that $(\xi, \eta)$ has a Gaussian distribution as well. Let $(H,[\cdot, \cdot])$ be a Hilbert space. If $f: X \rightarrow H$ and $g: Y \rightarrow H$ are such that $f(\xi), g(\eta) \in L^{2}(\Omega ; H)$ and $\mathbb{E} f(\xi)=\mathbb{E} g(\eta)=0$, then

$$
\left|\mathbb{E}[f(\xi), g(\eta)]_{H}\right| \leq \rho\|f(\xi)\|_{L^{2}(\Omega ; H)}\|g(\eta)\|_{L^{2}(\Omega ; H)},
$$

where $\rho$ is as in Eq. 3.1. Moreover, if $f$ or $g$ is even, then

$$
|\mathbb{E} f(\xi) g(\eta)| \leq \rho^{2}\|f(\xi)\|_{L^{2}(\Omega ; H)}\|g(\eta)\|_{L^{2}(\Omega ; H)} .
$$

Proof The constant $C$ appearing in Proposition 3.1 and Theorem 3.4 comes from estimate (3.5). Therefore, it suffices to show that

$$
\left\|Q_{H}^{\mu}(t) f\right\|_{L^{2}(\mu ; H)} \leq e^{-t}\|f\|_{L^{2}(\mu ; H)},
$$

where $\mu$ and $f$ are as in Proposition 3.1 and similarly with $e^{-t}$ replaced by $e^{-2 t}$ if $f$ is also even. The case that $H=\mathbb{R}$ follows from [23, Lemma 1.4.1]. In the general case we may assume without loss of generality that $H$ is separable, because $f(\xi)$ and $g(\eta)$ are strongly measurable. Let $\left(h_{n}\right)_{n \geq 1}$ be an orthonormal basis for $H$. By the result for the case $H=\mathbb{R}$ we obtain that

$$
\begin{aligned}
\left\|Q_{H}^{\mu}(t) f\right\|_{L^{2}(\mu ; H)}^{2} & =\sum_{n \geq 1} \int_{X}\left|Q^{\mu}(t)\left[f, h_{n}\right]\right|^{2} d \mu \\
& \leq e^{-2 t} \sum_{n \geq 1} \int_{X}\left|\left[f, h_{n}\right]\right|^{2} d \mu=e^{-2 t}\|f\|_{L^{2}(\mu ; H)}^{2} .
\end{aligned}
$$

For the improvement in the case $f$ is even, one can argue in the same way.

\section{Sequences of Gaussian Random Variables}

In this section we will consider sequences of centered multivariate Gaussian Radon random variables $\left(\xi_{n}\right)_{n \geq 1}$ with values in a Banach space $X$. Here multivariate means that $\left(\xi_{n}\right)_{n=1}^{N}$ is a Gaussian random variable in $X^{N}$ for each $N$. Many of the results below extend without difficulty to the more general setting where each $\xi_{n}$ takes values in a Banach space $X_{n}$, and $\left(\xi_{n}\right)_{n=1}^{N}$ is a Gaussian random variable in $\bigotimes_{n=1}^{N} X_{n}$. This can easily be checked by the interested reader.

Let $B_{X^{*}}$ be the unit ball of $X^{*}$. Let $\sigma_{n}=\sup _{x^{*} \in B_{X^{*}}}\left(\mathbb{E}\left\langle\xi_{n}, x^{*}\right\rangle^{2}\right)^{\frac{1}{2}}$ denote the weak variance of $\xi_{n}$. Define the correlation matrix $\rho_{i j}$ of $\left(\xi_{i}\right)_{i \geq 1}$ by $\rho_{i j}=\rho\left(\xi_{i}, \xi_{j}\right)$, where $\rho$ is as in Eq. 3.1. Throughout this section we will assume that

$$
C:=\sup _{i} \sum_{j \geq 1}\left|\rho_{i j}\right|<\infty \text {. }
$$

If $X=\mathbb{R}$, then Eq. 4.1 reduces to the assumption in [2]. Note that since $\rho_{i i}=1$, one always has $C \geq 1$. The $\left(\xi_{n}\right)_{n \geq 1}$ are pairwise independent if and only if $C=1$. 
With the same argument as in [2] one can obtain the following extension of $[2$, Lemma 2.1] to the vector valued setting.

Lemma 4.1 Under the assumption (4.1) for arbitrary Borel subsets $\left(A_{i}\right)_{i \geq 1}$ of $X$ we have

$$
\mathbb{E}\left|\frac{\sum_{i=1}^{n} \mathbf{1}_{A_{i}}\left(\xi_{i}\right)}{\sum_{i=1}^{n} \mathbb{P}\left(\xi_{i} \in A_{i}\right)}-1\right|^{2} \leq \frac{C}{\sum_{i=1}^{n} \mathbb{P}\left(\xi_{i} \in A_{i}\right)}
$$

Therefore, also the same Borel-Cantelli lemma [2, Corollary 2.1] extends to the vector-valued setting.

Corollary 4.2 Assume Eq. 4.1. If $\left(A_{i}\right)_{i \geq 1}$ is a sequence of Borel sets such that $\sum_{i=1}^{\infty} \mathbb{P}\left(\xi_{i} \in A_{i}\right)=\infty$, then $\mathbb{P}\left(\xi_{i} \in A_{i}\right.$ i.o. $)=1$. Moreover, if $\sum_{i=1}^{\infty} \mathbb{P}\left(\xi_{i} \in A_{i}\right)<\infty$, then $\mathbb{P}\left(\xi_{i} \in A_{i}\right.$ i.o. $)=0$.

Of course the second statement holds without assumption (4.1). As a consequence we obtain the following result (see [2, Corollary 2.4]).

Corollary 4.3 Assume Eq. 4.1. Then the following statements hold:

(1) there exists an $r \geq 0$ such that $\sum_{i=1}^{\infty} \mathbb{P}\left(\left\|\xi_{i}\right\|>r\right)<\infty$ if and only if $\mathbb{P}\left(\sup _{i \geq 1}\left\|\xi_{i}\right\|<\infty\right)=1$.

(2) for all $r \geq 0, \sum_{i=1}^{\infty} \mathbb{P}\left(\left\|\xi_{i}\right\|>r\right)<\infty$ if and only if $\mathbb{P}\left(\lim _{i \rightarrow \infty}\left\|\xi_{i}\right\|=0\right)=1$.

If $\mathbb{P}\left(\sup _{i \geq 1}\left\|\xi_{i}\right\|<\infty\right)=1$, then $\left(\xi_{i}\right)_{i \geq 1}$ can be seen as a Gaussian random variable in $l^{\infty}(X)$ with a definition of a Gaussian random variable which is slightly more general (cf. [20] or Section 5). In particular, $\mathbb{E} \sup _{n}\left\|\xi_{n}\right\|<\infty$. In [15] two-sided estimates for $\mathbb{E} \sup _{n}\left\|\xi_{n}\right\|$ have been found under the condition that the $\xi_{n}$ are independent. Below we extend this result under the assumption that Eq. 4.1 holds.

Let $\Theta: \mathbb{R} \rightarrow \mathbb{R}$ be defined as $\Theta(x)=x^{2} e^{-\frac{1}{x^{2}}}$, let $l^{\Theta}$ denote the Orlicz sequence space associated to $\Theta$ and let $\rho_{\Theta}(a)$ denote the Luxemburg norm of a sequence $a=\left(a_{n}\right)_{n \geq 1}$, i.e.

$$
\rho_{\Theta}(a):=\inf \left\{\delta>0: \sum_{n \geq 1} \frac{a_{n}^{2}}{\delta^{2}} \exp \left(-\frac{\delta^{2}}{2 a_{n}^{2}}\right) \leq 1\right\}<\infty .
$$

Theorem 4.4 Let $X$ be a Banach space. Let $\left(\xi_{n}\right)_{n \geq 1}$ be an $X$-valued centered multivariate Gaussian Radon random variables with first moments $\left(m_{n}\right)_{n \geq 1}$ and weak variances $\left(\sigma_{n}\right)_{n \geq 1}$. Let $m=\sup _{n \geq 1} m_{n}$. Assume that condition (4.1) holds. Then

$$
\max \left\{m, \frac{1}{3 C} \rho_{\Theta}\left(\left(\sigma_{n}\right)_{n \geq 1}\right)\right\} \leq \mathbb{E} \sup _{n \geq 1}\left\|\xi_{n}\right\| \leq m+3 \rho_{\Theta}\left(\left(\sigma_{n}\right)_{n \geq 1}\right) .
$$

The upper estimate of $\mathbb{E} \sup _{n \geq 1}\left\|\xi_{n}\right\|$ in Theorem 4.4 has been obtained in [15] without any assumption on the correlation structure. The lower estimate with $C=1$ has been proved there in the case where the $\left(\xi_{n}\right)_{n \geq 1}$ are independent. 
Proof To prove the lower estimate, we extend the arguments from [15]. Note that $\mathbb{E} \sup _{n \geq 1}\left\|\xi_{n}\right\| \geq m$ is clear. As for the estimate for $\rho_{\Theta}\left(\left(\sigma_{n}\right)_{n \geq 1}\right)$, by scaling we may assume that $\mathbb{E} \sup _{n \geq 1}\left\|\xi_{n}\right\|=1$. Let $r>1$ be arbitrary. Then $\mathbb{P}\left(\sup _{n \geq 1}\left\|\xi_{n}\right\|>r\right) \leq 1 / r$. Let $\varepsilon \in(0,1)$ be arbitrary. For each $j$ choose $x_{j}^{*} \in B_{X^{*}}$ such that $\left(\mathbb{E}\left\langle\xi_{j}, x_{j}^{*}\right\rangle^{2}\right)^{\frac{1}{2}} \geq$ $\sigma_{j}(1-\varepsilon)$. Let $X_{j}=\frac{\left\langle\xi_{j}^{*}, x_{j}^{*}\right\rangle}{\left(\mathbb{E}\left\langle\xi_{j}^{*}, x_{j}^{*}\right)^{2}\right)^{\frac{1}{2}}}$. Then with $r_{j}=r /\left(\mathbb{E}\left\langle\xi_{j}^{*}, x_{j}^{*}\right\rangle^{2}\right)^{\frac{1}{2}}$ it follows from Eq. 4.1 and $[2$, Lemma 2.1] (or Eq. 4.2 in the real case) that

$$
\sum_{j=1}^{n} \mathbb{P}\left(\left|X_{j}\right| \geq r_{j}\right) \leq \frac{C}{\mathbb{E}\left|\frac{\sum_{j=1}^{n} 1_{\left|X_{i}\right| \geq r_{j}}}{\sum_{j=1}^{n} \mathbb{P}\left(\left|X_{j}\right| \geq r_{j}\right)}-1\right|^{2}} .
$$

For all $n \geq 1$, we have

$$
\begin{aligned}
\mathbb{E}\left|\frac{\sum_{j=1}^{n} 1_{\left|X_{i}\right| \geq r_{j}}}{\sum_{j=1}^{n} \mathbb{P}\left(\left|X_{j}\right| \geq r_{j}\right)}-1\right|^{2} & \geq \mathbb{E}\left[|-1|^{2} ; \bigcap_{j \geq 1}\left\{\left|X_{j}\right|<r_{j}\right\}\right] \\
& =1-\mathbb{P}\left(\bigcup_{j \geq 1}\left\{\left|X_{j}\right| \geq r_{j}\right\}\right) \\
& \geq 1-\mathbb{P}\left(\sup _{j \geq 1}\left|\left\langle\xi_{j}, x_{j}^{*}\right\rangle\right| \geq r\right) \\
& \geq 1-\mathbb{P}\left(\sup _{j \geq 1}\left\|\xi_{j}\right\| \geq r\right) \geq 1-\frac{1}{r} .
\end{aligned}
$$

If we combine this with Eq. 4.3 we obtain that

$$
\sum_{j \geq 1} \mathbb{P}\left(\left|X_{j}\right| \geq r_{j}\right) \leq \frac{C}{1-\frac{1}{r}} .
$$

On the other hand since $\mathbb{E}\left\langle\xi_{j}, x_{n_{j}}^{*}\right\rangle^{2} \geq(1-\varepsilon)^{2} \sigma_{j}^{2}$,

$$
\begin{aligned}
\mathbb{P}\left(\left|X_{j}\right| \geq r_{j}\right) & \geq \frac{\sqrt{2}}{\sqrt{\pi}} \int_{r /\left(\sigma_{j}(1-\varepsilon)\right)} e^{-t^{2} / 2} d t \\
& \geq \frac{\sqrt{2}}{\sqrt{\pi}} \frac{r \sigma_{j}(1-\varepsilon)}{\sigma_{j}^{2}(1-\varepsilon)^{2}+r^{2}} \exp \left(-\frac{1}{2}\left(\frac{r}{\sigma_{j}(1-\varepsilon)}\right)^{2}\right) .
\end{aligned}
$$

Since $\varepsilon \in(0,1)$ was arbitrary it follows that

$$
\begin{aligned}
\frac{C}{1-\frac{1}{r}} & \geq \frac{r \sqrt{2}}{\sqrt{\pi}} \sum_{j \geq 1} \frac{\sigma_{j}}{\sigma_{j}^{2}+r^{2}} \exp \left(-\frac{1}{2}\left(\frac{r}{\sigma_{j}}\right)^{2}\right) \\
& \geq \frac{r^{3} \sqrt{2}}{\sqrt{\pi}} \frac{1}{\left(\frac{\pi}{2}\right)^{\frac{3}{2}}+r^{2}\left(\frac{\pi}{2}\right)^{\frac{1}{2}}} \sum_{j \geq 1}\left(\frac{\sigma_{j}}{r}\right)^{2} \exp \left(-\frac{1}{2}\left(\frac{r}{\sigma_{j}}\right)^{2}\right),
\end{aligned}
$$


where we used

$$
\sigma_{j}^{2}=\sup _{x^{*} \in B_{X^{*}}} \mathbb{E}\left\langle\xi_{j}, x^{*}\right\rangle^{2}=\frac{\pi}{2} \sup _{x^{*} \in B_{X^{*}}}\left(\mathbb{E}\left|\left\langle\xi_{j}, x^{*}\right\rangle\right|\right)^{2} \leq \frac{\pi}{2}\left(\mathbb{E}\left\|\xi_{j}\right\|\right)^{2} \leq \frac{\pi}{2}
$$

Therefore,

$$
\sum_{j \geq 1}\left(\frac{\sigma_{j}}{r}\right)^{2} \exp \left(-\frac{1}{2}\left(\frac{r}{\sigma_{j}}\right)^{2}\right) \leq \frac{C \sqrt{\pi}\left(\left(\frac{\pi}{2}\right)^{\frac{3}{2}}+r^{2}\left(\frac{\pi}{2}\right)^{\frac{1}{2}}\right)}{\sqrt{2}\left(r^{3}-r\right)} .
$$

Considering $r \geq 2$ only, one can estimate

$$
\frac{C \sqrt{\pi}\left(\left(\frac{\pi}{2}\right)^{\frac{3}{2}}+r^{2}\left(\frac{\pi}{2}\right)^{\frac{1}{2}}\right)}{\sqrt{2}\left(r^{3}-r\right)} \leq \frac{C \sqrt{\pi}\left(\left(\frac{\pi}{2}\right)^{\frac{3}{2}} r^{2} / 4+r^{2}\left(\frac{\pi}{2}\right)^{\frac{1}{2}}\right)}{\sqrt{2}\left(r^{3}-r\right)} \leq \frac{C \sqrt{\pi}\left(\left(\frac{\pi}{2}\right)^{\frac{3}{2}} \frac{1}{4}+\left(\frac{\pi}{2}\right)^{\frac{1}{2}}\right)}{\sqrt{2}\left(r-\frac{1}{2}\right)} .
$$

Noting that $C \geq 1$, one easily checks that the latter expression is less than 1 for $r=$ $3 C$. This completes the proof.

In the last part of this section we will consider several cases of the law of large numbers for random variables $\left(f_{n}\left(\xi_{n}\right)\right)_{n \geq 1}$, where the $\left(f_{n}\right)_{n \geq 1}$ are measurable functions from the locally convex space $X$ into a Banach space $Y$. The presented results generalize several results from $[1,2]$, where the case that $X=Y=\mathbb{R}$ is studied.

First we state a generalization of [1, Theorem 3.1].

Theorem 4.5 Let $\mu$ be a centered Gaussian Radon measure on a locally convex space $X$. Let $(H,[\cdot, \cdot])$ be a separable Hilbert space. Let $\left(\xi_{n}\right)_{n \geq 1}$ be a multivariate Gaussian sequence with distribution $\mu$. Let $\left(f_{n}\right)_{n \geq 1}$ be Borel measurable functions from $X$ into $H$ such $\left(\left\|f_{n}\right\|\right)_{n \geq 1}$ are uniformly integrable in $L^{1}(\mu)$. If Eq. 4.1 holds, then

$$
\lim _{n \rightarrow \infty} \frac{1}{n} \sum_{i=1}^{n}\left(f_{i}\left(\xi_{i}\right)-\mathbb{E} f_{i}\left(\xi_{i}\right)\right)=0 \text { in } L^{1}(\Omega ; H) .
$$

Using Corollary 3.5 the proof is a straightforward extension of the proof from [1, Theorem 3.1]. We leave the details to the reader.

A similar generalization holds for [1, Theorem 3.2] as the interested reader can easily check. Next we extend [2, Theorem 3.3] to the vector-valued setting.

Theorem 4.6 Let $\mu$ be a centered Gaussian Radon measure on a locally convex space $X$. Let $Y$ be a Banach space. Let $\left(\xi_{n}\right)_{n \geq 1}$ be a multivariate Gaussian sequence with distribution $\mu$. Let $f \in L^{1}(\mu ; Y)$. If Eq. 4.1 holds, then

$$
\lim _{n \rightarrow \infty} \frac{1}{n} \sum_{i=1}^{n} f\left(\xi_{i}\right)=\mathbb{E} f\left(\xi_{1}\right) \text { a.s. and in } L^{1} .
$$

In particular, if $X$ is a separable Banach space, then

$$
\lim _{n \rightarrow \infty} \frac{1}{n} \sum_{i=1}^{n} \xi_{i}=0 \text { a.s. and in } L^{p} \text { for all } p \in[1, \infty)
$$


Proof The proof of the a.s. convergence is divided into two steps.

Step 1: The case $Y=\mathbb{R}$. This can be proved in the same way as [2, Theorem 3.3] using the straightforward extension of [2, Lemma 3.2] or Lemma 4.8 with $H=\mathbb{R}$.

Step 2: The general case. We use a well-known approximation argument (cf. [14, Theorem III.1.1]). We present it for convenience to the reader. Without loss of generality we may assume $\mathbb{E} f\left(\xi_{1}\right)=0$. Let $\varepsilon>0$ be arbitrary. Since $f \in L^{1}(\mu ; Y)$ we can find a simple Borel function $g: X \rightarrow Y$ such that

$$
\int_{X} g(x) d \mu(x)=0 \text { and }\|f-g\|_{L^{1}(\mu ; Y)}<\varepsilon .
$$

Since $g(X) \subset Y$ is finite dimensional, we can apply Step 1 (on each coordinate) to obtain

$$
\lim _{n \rightarrow \infty} \frac{1}{n} \sum_{i=1}^{n} g\left(\xi_{i}\right)=0 \text { a.s. }
$$

Let $h: X \rightarrow \mathbb{R}$ be defined as $h(x)=\|f(x)-g(x)\|$. Again by Step 1,

$$
\lim _{n \rightarrow \infty} \frac{1}{n} \sum_{i=1}^{n} h\left(\xi_{i}\right)=\mathbb{E} h\left(\xi_{1}\right)<\varepsilon \text { a.s. }
$$

We conclude that

$$
\limsup _{n \rightarrow \infty}\left\|\frac{1}{n} \sum_{i=1}^{n} f\left(\xi_{i}\right)\right\| \leq \limsup _{n \rightarrow \infty}\left(\left\|\frac{1}{n} \sum_{i=1}^{n} g\left(\xi_{i}\right)\right\|+\left\|\frac{1}{n} \sum_{i=1}^{n} h\left(\xi_{i}\right)\right\|\right) \leq \varepsilon \text { a.s. }
$$

Since $\varepsilon>0$ is arbitrary, the result follows.

The final statement follows by taking $f(x)=x$, and noting that by the separability of $X$, the function $f$ is strongly measurable. By [26] the $L^{p}$-convergence follows from the almost sure convergence.

The proof of the $L^{1}$ convergence follows by a similar approximation result and [1, Theorem 3.1].

Conversely, we have the following result which extends [2, Proposition 3.1].

Proposition 4.7 Let $\mu$ be a centered Gaussian Radon measure on a locally convex space $X$. Let $Y$ be a Banach space. Let $\left(\xi_{n}\right)_{n \geq 1}$ be a multivariate Gaussian sequence with distribution $\mu$. Assume Eq. 4.1 holds. If $\limsup _{n \rightarrow \infty}\left\|n^{-1} \sum_{i=1}^{n} f\left(\xi_{i}\right)\right\|<\infty$ on a set of positive probability, then $f\left(\xi_{1}\right) \in L^{1}(\mu ; Y)$.

Using Corollary 4.2 one can show the result in the same as in [2].

Next, we consider the case of a.s. convergence where the $\left(\xi_{i}\right)_{i \geq 1}$ are not necessarily identically distributed and the function $f$ is replaced by a sequence of functions $\left(f_{i}\right)_{i \geq 1}$. For this we will need the following lemma which extends [2, Lemma 3.2]. For a random variable $\xi: \Omega \rightarrow X$, where $X$ is a Banach space, let $\operatorname{Var}(\xi)=\mathbb{E}\|\xi-\mathbb{E} \xi\|^{2}$. 
Lemma 4.8 Let $X$ be a locally convex space. Let $(H,[\cdot, \cdot])$ be a separable Hilbert space. Let $\left(\xi_{n}\right)_{n \geq 1}$ be a centered multivariate Gaussian Radon sequence that satisfies Eq. 4.1. Let $\left(f_{n}\right)_{n \geq 1}$ be Borel measurable functions from $X$ into $H$. Then for each $n \geq 1$, we have

$$
C^{-1} \sum_{i=1}^{n} \operatorname{Var}\left(f_{i}\left(\xi_{i}\right)\right) \leq \operatorname{Var}\left(\sum_{i=1}^{n} f_{i}\left(\xi_{i}\right)\right) \leq C \sum_{i=1}^{n} \operatorname{Var}\left(f_{i}\left(\xi_{i}\right)\right) .
$$

The first inequality seems to be new even in the case $X=H=\mathbb{R}$.

Proof Without loss of generality, we can assume $\mathbb{E} f_{i}\left(\xi_{i}\right)=0$ for all $i$. By Corollary 3.5, the Cauchy-Schwarz inequality and [2, Lemma 3.1],

$$
\begin{aligned}
\operatorname{Var}\left(\sum_{i=1}^{n} f_{i}\left(\xi_{i}\right)\right) & =\sum_{i, j=1}^{n} \mathbb{E}\left[f_{i}\left(\xi_{i}\right), f_{j}\left(\xi_{i}\right)\right] \leq \sum_{i, j=1}^{n} \rho_{i j}\left(\operatorname{Var}\left(f_{i}\left(\xi_{i}\right)\right)\right)^{\frac{1}{2}}\left(\operatorname{Var}\left(f_{j}\left(\xi_{j}\right)\right)\right)^{\frac{1}{2}} \\
& \leq\left(\sum_{j=1}^{n}\left|\sum_{i=1}^{n} \rho_{i j}\left(\operatorname{Var}\left(f_{i}\left(\xi_{i}\right)\right)\right)^{\frac{1}{2}}\right|^{2}\right)^{\frac{1}{2}}\left(\sum_{j=1}^{n} \operatorname{Var}\left(f_{j}\left(\xi_{j}\right)\right)\right)^{\frac{1}{2}} \\
& \leq C \sum_{j=1}^{n} \operatorname{Var}\left(f_{j}\left(\xi_{j}\right)\right) .
\end{aligned}
$$

This proves the second inequality.

To prove the first inequality let $\left(\hat{\rho}_{i j}\right)$ be defined as $\hat{\rho}_{i j}=\rho_{i j}$ if $i \neq j$ and $\hat{\rho}_{i i}=0$. As in [2, Lemma 3.1] by the assumption (4.1), $\|\hat{\rho}\|_{\mathcal{B}\left(\ell^{2}\right)}=C-1$. Recall that $C \geq 1$. We obtain

$$
\begin{aligned}
\left|\sum_{i \geq 1} \sum_{j \neq i} \mathbb{E}\left[f_{i}\left(\xi_{i}\right), f_{j}\left(\xi_{j}\right)\right]\right| & \leq \sum_{i \geq 1} \sum_{j \neq i} \hat{\rho}_{i j}\left(\operatorname{Var}\left(f_{i}\left(\xi_{i}\right)\right)\right)^{\frac{1}{2}}\left(\operatorname{Var}\left(f_{j}\left(\xi_{j}\right)\right)\right)^{\frac{1}{2}} \\
& \leq(C-1) \sum_{i \geq 1} \operatorname{Var}\left(f_{i}\left(\xi_{i}\right)\right) .
\end{aligned}
$$

Therefore, it follows that

$$
\begin{aligned}
\sum_{i=1}^{n} \operatorname{Var}\left(f_{i}\left(\xi_{i}\right)\right) & =C \sum_{i=1}^{n} \operatorname{Var}\left(f_{i}\left(\xi_{i}\right)\right)-(C-1) \sum_{i=1}^{n} \operatorname{Var}\left(f_{i}\left(\xi_{i}\right)\right) \\
& \leq C \sum_{i=1}^{n} \operatorname{Var}\left(f_{i}\left(\xi_{i}\right)\right)+\sum_{i \geq 1} \sum_{j \neq i} \mathbb{E}\left[f_{i}\left(\xi_{i}\right), f_{j}\left(\xi_{j}\right)\right] \\
& \leq C \operatorname{Var}\left(\sum_{i=1}^{n} f_{i}\left(\xi_{i}\right)\right) .
\end{aligned}
$$

The next result is a generalization of [2, Theorem 3.6]. 
Theorem 4.9 Let $X$ be a locally convex space and let $(H,[\cdot, \cdot])$ be a separable Hilbert space. Let $\left(\xi_{n}\right)_{n \geq 1}$ be a centered multivariate Gaussian Radon sequence in $X$ that satisfies Eq. 4.1. Let $f_{n}: X \rightarrow H$ be such $f_{n}\left(\xi_{n}\right) \in L^{2}(\Omega ; H)$ for all $n \geq 1$. Moreover, assume

$$
\sup _{n \geq 1} \mathbb{E}\left\|f_{n}\left(\xi_{n}\right)\right\|<\infty \text { and } \sum_{i \geq 1} \frac{\operatorname{Var}\left(f_{i}\left(\xi_{i}\right)\right)}{i^{2}}<\infty \text {. }
$$

Then

$$
\lim _{n \rightarrow \infty} \frac{1}{n} \sum_{i=1}^{n} f_{i}\left(\xi_{i}\right)-\mathbb{E} f_{i}\left(\xi_{i}\right)=0 \text { a.s. }
$$

If additionally, $X=H$ and $\sup _{n \geq 1} \mathbb{E}\left\|\xi_{n}\right\|<\infty$, then

$$
\lim _{n \rightarrow \infty} \frac{1}{n} \sum_{i=1}^{n} \xi_{i}=0 \text { a.s. and in } L^{p} \text { for all } p<\infty \text {. }
$$

The proof of [2, Theorem 3.6] is based on the techniques of $[10,11]$ which use the order of $\mathbb{R}$. Since we consider vector-valued random variables, we have to extend this argument.

Proof Let $\left(h_{l}\right)_{l \geq 1}$ be an orthonormal basis for $H$. For an $h \in H$, let $(h)^{+}$and $(h)^{-}$be defined as

$$
(h)^{ \pm}=\sum_{l \geq 1}\left[h, h_{l}\right]^{ \pm} h_{l},
$$

where for $a \in \mathbb{R}, a^{+}=\max \{a, 0\}$ and $a^{-}=\max \{-a, 0\}$. With this notation we can write

$$
f_{i}\left(X_{i}\right)-\mathbb{E} f_{i}\left(X_{i}\right)=\left(f_{i}\left(X_{i}\right)-\mathbb{E} f_{i}\left(X_{i}\right)\right)^{+}-\left(f_{i}\left(X_{i}\right)-\mathbb{E} f_{i}\left(X_{i}\right)\right)^{-} .
$$

Since $\left[(h)^{+},(h)^{-}\right]=0$, one has

$$
\left\|f_{i}\left(X_{i}\right)-\mathbb{E} f_{i}\left(X_{i}\right)\right\|^{2}=\left\|\left(f_{i}\left(X_{i}\right)-\mathbb{E} f_{i}\left(X_{i}\right)\right)^{+}\right\|^{2}+\left\|\left(f_{i}\left(X_{i}\right)-\mathbb{E} f_{i}\left(X_{i}\right)\right)^{-}\right\|^{2}
$$

and therefore,

$$
\operatorname{Var}\left(f_{i}\left(X_{i}\right)\right) \geq \operatorname{Var}\left(\left(f_{i}\left(X_{i}\right)-\mathbb{E} f_{i}\left(X_{i}\right)\right)^{+}\right)+\operatorname{Var}\left(\left(f_{i}\left(X_{i}\right)-\mathbb{E} f_{i}\left(X_{i}\right)\right)^{-}\right) .
$$

It follows that it suffices to prove the theorem in the case that $\left[f_{i}\left(X_{i}\right), h_{l}\right] \geq 0$ for all $l \geq 1$ and all $i \geq 1$.

As in [2] let $\alpha>1$ be fixed and define the integers $\left(k_{n}\right)_{n \geq 1}$ by $k_{0}=1$ and $k_{n}=\left[\alpha^{n}\right]$, where $[x]$ is the greatest integer less than or equal to $x$. Then $\lim _{n \rightarrow \infty} \frac{k_{n}}{k_{n+1}}=\frac{1}{\alpha}$ and for all $m \geq 1$ there exists an $n(m) \geq 1$ such that $k_{n(m)-1} \leq m \leq k_{n(m)}$. We claim that for all $\alpha>1$,

$$
\lim _{n \rightarrow \infty} \frac{S_{k_{n}}-\mathbb{E} S_{k_{n}}}{k_{n}}=0 \text { a.s. }
$$


Before we prove the claim let us show how the theorem follows from this. Since

$$
\begin{aligned}
\left\|S_{m}-\mathbb{E} S_{m}\right\|^{2}= & \sum_{l \geq 1} \sum_{i, j=1}^{m}\left[f_{i}\left(X_{i}\right)-\mathbb{E} f_{i}\left(X_{i}\right), h_{l}\right]\left[f_{j}\left(X_{j}\right)-\mathbb{E} f_{j}\left(X_{j}\right), h_{l}\right] \\
\leq & \sum_{l \geq 1} \sum_{i, j=1}^{k_{n(m)-1}}\left[f_{i}\left(X_{i}\right)-\mathbb{E} f_{i}\left(X_{i}\right), h_{l}\right]\left[f_{j}\left(X_{j}\right)-\mathbb{E} f_{j}\left(X_{j}\right), h_{l}\right] \\
& +\sum_{l \geq 1} \sum_{i, j=k_{n(m)-1}+1}^{k_{n(m)}}\left(\left[f_{i}\left(X_{i}\right), h_{l}\right]\left[f_{j}\left(X_{j}\right), h_{l}\right]\right. \\
& \left.+\left[\mathbb{E} f_{i}\left(X_{i}\right), h_{l}\right]\left[\mathbb{E} f_{j}\left(X_{j}\right), h_{l}\right]\right) \\
= & \left\|S_{k_{n(m)-1}}-\mathbb{E} S_{k_{n(m)-1}}\right\|^{2}+\left\|\sum_{i=k_{n(m)-1}+1}^{k_{n(m)}} f_{i}\left(X_{i}\right)\right\|^{2}+\left\|\sum_{i=k_{n(m)-1}+1}^{k_{n(m)}} \mathbb{E} f_{i}\left(X_{i}\right)\right\|^{2},
\end{aligned}
$$

it follows that

$$
\frac{\left\|S_{m}-\mathbb{E} S_{m}\right\|}{m} \leq \frac{\left\|S_{k_{n(m)-1}}-\mathbb{E} S_{k_{n(m)-1}}\right\|}{k_{n_{m}-1}}+2 \sup _{i \geq 1} \mathbb{E}\left\|f_{i}\left(X_{i}\right)\right\| \frac{k_{n(m)}-k_{n(m)-1}}{k_{n(m)-1}} .
$$

We obtain

$$
\limsup _{m \rightarrow \infty} \frac{\left\|S_{m}-\mathbb{E} S_{m}\right\|}{m} \leq 2 \sup _{i \geq 1} \mathbb{E}\left\|f_{i}\left(X_{i}\right)\right\|(\alpha-1) .
$$

Since $\alpha>1$ is arbitrary, this gives the result.

It remains to show Eq. 4.6. Equivalently, we can show that $\mathbb{P}\left(\lim \sup _{n \rightarrow \infty} \| S_{k_{n}}-\right.$ $\left.\mathbb{E} S_{k_{n}} \|>\varepsilon k_{n}\right)=0$ for all $\varepsilon>0$. For this it suffices to show that for all $\varepsilon>0$,

$$
\sum_{n \geq 1} \mathbb{P}\left(\left\|S_{k_{n}}-\mathbb{E} S_{k_{n}}\right\|>\varepsilon k_{n}\right)<\infty .
$$

Let $\varepsilon>0$ be arbitrary. By Chebychev's inequality and by Lemma 4.8 it follows that

$$
\begin{aligned}
\sum_{n \geq 1} \mathbb{P} & \left(\left\|S_{k_{n}}-\mathbb{E} S_{k_{n}}\right\|>\varepsilon k_{n}\right) \leq \frac{1}{\varepsilon^{2}} \sum_{n \geq 1} \frac{\operatorname{Var}\left(S_{k_{n}}\right)}{k_{n}^{2}} \\
& \leq \frac{C}{\varepsilon^{2}} \sum_{n \geq 1} \frac{1}{k_{n}^{2}} \sum_{i=1}^{k_{n}} \operatorname{Var}\left(f_{i}\left(\xi_{i}\right)\right)=\frac{C}{\varepsilon^{2}} \sum_{i \geq 1} \operatorname{Var}\left(f_{i}\left(\xi_{i}\right)\right) \sum_{n \geq 1, i \leq k_{n}} \frac{1}{k_{n}^{2}} \\
& \leq \frac{C C_{\alpha}}{\varepsilon^{2}} \sum_{i \geq 1} \frac{\operatorname{Var}\left(f_{i}\left(\xi_{i}\right)\right)}{i^{2}}<\infty .
\end{aligned}
$$

The final result follows from the first part and [26].

One could hope to extend Theorem 4.9 to more general Banach spaces. For instance under assumption on the type of the Banach space. We only know how to extend the second part of Theorem 4.9 to Banach spaces with non-trivial type. For the definition and properties of type we refer to [14]. We will need the following proposition. 
Proposition 4.10 Let $X$ be a Banach space. Let $\left(\xi_{n}\right)_{n \geq 1}$ be a centered multivariate Gaussian Radon sequence in $X$ that satisfies Eq. 4.1. Let $\left(\tilde{\xi}_{n}\right)_{n \geq 1}$ be a sequence of independent Gaussian Radon random variables such that for each $n \geq 1, \tilde{\xi}_{n} \stackrel{D}{=} \xi_{i}$. Let $\varphi: X \rightarrow \mathbb{R}_{+}$be a measurable convex function. Then for all $n \geq 1$,

$$
\mathbb{E} \varphi\left(C^{-\frac{1}{2}} \sum_{i=1}^{n} \tilde{\xi}_{i}\right) \leq \mathbb{E} \varphi\left(\sum_{i=1}^{n} \xi_{i}\right) \leq \mathbb{E} \varphi\left(C^{\frac{1}{2}} \sum_{i=1}^{n} \tilde{\xi}_{i}\right)
$$

In particular, $\sum_{i>1} \tilde{\xi}_{i}$ converges in $L^{2}(\Omega ; X)$ if and only if $\sum_{i>1} \xi_{i}$ converges in $L^{2}(\Omega ; X)$.

As explained in [19, p. 129], by Anderson's inequality it also holds that for all convex and symmetric Borel sets $K \subset X$, one has

$$
\mathbb{P}\left(C^{-\frac{1}{2}} \sum_{i=1}^{n} \tilde{\xi}_{i} \notin K\right) \leq \mathbb{P}\left(\sum_{i=1}^{n} \xi_{i} \notin K\right) \leq \mathbb{P}\left(C^{\frac{1}{2}} \sum_{i=1}^{n} \tilde{\xi}_{i} \notin K\right)
$$

Proof Let $\xi=\sum_{i=1}^{n} \xi_{i}$ and $\tilde{\xi}=\sum_{i=1}^{n} \tilde{\xi}_{i}$. First we show the right-hand side of Eq. 4.7. One can check that for all $x^{*} \in X^{*}, \mathbb{E}\left\langle\xi, x^{*}\right\rangle^{2} \leq C \mathbb{E}\left\langle\tilde{\xi}, x^{*}\right\rangle^{2}$. Indeed, this follows from Lemma 4.8 with $H=\mathbb{R}$ and

$$
\mathbb{E}\left\langle\tilde{\xi}, x^{*}\right\rangle^{2}=\sum_{i=1}^{n} \mathbb{E}\left\langle\tilde{\xi}_{i}, x^{*}\right\rangle^{2}
$$

Now the result follows from a standard argument based on covariance domination. We present it for convenience of the reader. By [19, p. 128] we can find a centered Gaussian Radon random variable $\eta$ independent of $\xi$ and such that $C^{\frac{1}{2}} \tilde{\xi}$ has the same distribution as $\xi+\eta$ and $\xi-\eta$. It follows from the convexity of $\varphi$ that

$$
\mathbb{E} \varphi(\xi)=\mathbb{E} \varphi\left(\frac{\xi+\eta}{2}+\frac{\xi-\eta}{2}\right) \leq \frac{1}{2} \mathbb{E} \varphi(\xi+\eta)+\frac{1}{2} \mathbb{E} \varphi(\xi-\eta)=\mathbb{E} \varphi\left(C^{\frac{1}{2}} \tilde{\xi}\right) .
$$

To prove the left-hand side of Eq. 4.7 note that it follows from Lemma 4.8 with $H=\mathbb{R}$ that $\mathbb{E}\left\langle\tilde{\xi}, x^{*}\right\rangle^{2} \leq C \mathbb{E}\left\langle\xi, x^{*}\right\rangle^{2}$. Now the result follows again by covariance domination.

The final statement clearly follows if we apply the result to $\varphi(x)=\|x\|^{2}$.

Theorem 4.11 Let $X$ be a Banach space with type $p$ for some $p>1$. Let $\left(\xi_{n}\right)_{n \geq 1}$ be a centered multivariate Gaussian Radon sequence in $X$ that satisfies Eq. 4.1 and $\sup _{n \geq 1} \mathbb{E}\left\|\xi_{n}\right\|<\infty$. Then

$$
\lim _{n \rightarrow \infty} \frac{1}{n} \sum_{i=1}^{n} \xi_{i}=0 \text { a.s. and in } L^{q} \text { for all } q<\infty
$$


Proof By the Kahane-Khinchine inequalities (cf. [20, Corollary 3.2]) and the assumption we have that $K:=\sup _{i>1} \mathbb{E}\left\|\xi_{i}\right\|^{p}<\infty$. By Proposition 4.10 applied to $\phi(x)=\|x\|^{p}$ and by the type $p$ condition we obtain that for all $n \geq 1$,

$$
\begin{aligned}
\mathbb{E}\left\|n^{-1} \sum_{i=1}^{n} \xi_{i}\right\|^{p} & \leq C^{\frac{p}{2}} C_{p}^{p} n^{-p} \sum_{i=1}^{n} \mathbb{E}\left\|\xi_{i}\right\|^{p} \\
& \leq C^{\frac{p}{2}} C_{p}^{p} K n^{-p+1} .
\end{aligned}
$$

For $q<\infty$ again by the Kahane-Khinchine inequalities it follows that

$$
\begin{aligned}
a_{q, n}:=\mathbb{E}\left\|n^{-1} \sum_{i=1}^{n} \xi_{i}\right\|^{q} & \leq C_{p, q}^{q}\left(\mathbb{E}\left\|n^{-1} \sum_{i=1}^{n} \xi_{i}\right\|^{p}\right)^{\frac{q}{p}} \\
& \leq C^{\frac{q}{2}} C_{p}^{q} K^{\frac{q}{p}} n^{\left(-1+\frac{1}{p}\right) q}
\end{aligned}
$$

Since $-1+\frac{1}{p}<0$, this proves the convergence in $L^{q}$ for arbitrary $q<\infty$. Furthermore, choosing $q<\infty$ so large that $\sum_{n \geq 1} a_{q, n}<\infty$, the Borel-Cantelli lemma implies that $\lim _{n \rightarrow \infty} n^{-1} \sum_{i=1}^{n} \xi_{i}=0$ a.s.

\section{Besov Regularity of Gaussian Processes}

Let $X$ be a Banach space. A process $G:[0,1] \times \Omega \rightarrow X$ is called a Gaussian process if it is strongly measurable and if for all $x^{*} \in X^{*}$, the real-valued process $\left\langle G, x^{*}\right\rangle$ is a Gaussian process.

A theorem of Kolmogorov (cf. [12, 25]) gives a way to obtain Hölder regularity of paths from Hölder regularity in $p$-th moments. In particular, if $G:[0,1] \times \Omega \rightarrow X$ is a Gaussian processes such that $G \in C^{\alpha}\left([0,1] ; L^{2}(\Omega ; X)\right)$ for some $\alpha \in(0,1)$, then $G$ is in $C^{\alpha}\left([0,1] ; L^{p}(\Omega ; X)\right)$ for all $p<\infty$. Applying the above mentioned theorem by Kolmogorov gives that $G$ has a version in $L^{p}\left(\Omega ; C^{\beta}([0,1] ; X)\right)$ for all $\beta \in(0, \alpha)$ and all $p<\infty$.

We will study regularity in the case $\beta=\alpha$. Of course it is not true in general that $G$ has a version with $\beta$-Hölder continuous paths. Indeed, if one takes $G=W$, where $W$ is a standard Brownian motion, then $W \in C^{\frac{1}{2}}\left([0,1] ; L^{2}(\Omega)\right)$, but a.s. $W \notin C^{\frac{1}{2}}([0,1])$ (cf. [25]). Instead of Hölder spaces one has to consider certain Besov spaces.

In [5] (also see [27]) it has been shown that $W \in B_{p, \infty}^{\frac{1}{2}}(0,1)$ a.s. for all $p<\infty$. Moreover, it has been shown in [6] that the paths of $W$ belong to a certain BesovOrlicz space. In [15] this has been extended to Brownian motions with values in a Banach space with more direct proofs.

In [7] the authors have obtained Besov and Besov-Orlicz regularity for fractional Brownian motion and other Gaussian processes. Their methods are based on nontrivial norm equivalences for Besov and Besov-Orlicz spaces. We will use the methods of [15] to obtain the Besov and Besov-Orlicz regularity directly from the definition of the spaces. We consider a certain class of vector-valued Gaussian processes which in particular contains fractional Brownian motion.

For random variables $\xi, \eta: \Omega \rightarrow X$ such that $(\xi, \eta)$ is a centered Gaussian random variables, we write $\rho(\xi, \eta)$ for the correlation number $\rho$ as defined in Eq. 3.1. 
Theorem 5.1 Let $X$ be a Banach space. Let $\alpha \in(0,1)$. Let $\left(G_{t}\right)_{t \in[0,1]}$ be an $X$-valued centered Gaussian process with the property that for all $s, t, a, b \in[0,1]$ with $s+a$, $t+a \in[0,1]$ and $(s, s+a) \cap(t, t+a)=\emptyset$, it holds that

$$
\rho(G(t+a)-G(t), G(s+a)-G(s)) \leq K(a,|t-s|),
$$

with $K:[0,1]^{2} \rightarrow[0,1]$ such that

$$
\sum_{n \geq 1} 2^{-2 n} \sum_{i=1}^{2^{n}-1}\left(2^{n}-i\right)\left(K\left(2^{-n}, i 2^{-n}\right)\right)^{2}<\infty
$$

If for some $p \in[1, \infty), G \in C^{\alpha}\left([0,1] ; L^{p}(\Omega ; X)\right)$, then for all $p \in[1, \infty), G \in$ $B_{p, \infty}^{\alpha}(0,1 ; X)$ a.s. Moreover, $G \in B_{\Phi_{2}, \infty}^{\alpha}(0,1 ; X)$ a.s.

If additionally,

$$
\liminf _{t \downarrow 0} t^{-\alpha p} \mathbb{E}\|G(t+s)-G(s)\|^{p} \geq k_{p}^{p},
$$

uniformly in $s \in[0,1)$ for some constant $k_{p}$ then $\|G\|_{B_{p, \infty}^{\alpha}(0,1 ; X)} \geq k_{p}$ a.s. and for all $q \in[1, \infty), G \notin B_{p, q}^{\alpha}(0,1 ; X)$ a.s.

If $G$ has independent increments Eq. 5.2 is always fulfilled because Eq. 5.1 holds with $K=0$. Secondly, for Eq. 5.2 it suffices to find $C, \varepsilon>0$ such that for all $a \in(0,1)$ and $b \geq 1, K(a, a b) \leq C b^{-\varepsilon}$. This condition should be compared to $[7$, p. 173 , Condition $(\mathrm{H})]$.

Proof The Kahane-Khinchine inequalities and the assumption yield that for all $p<\infty, c_{p}:=\|G\|_{C^{\alpha}\left([0,1] ; L^{p}(\Omega ; X)\right)}$ is of order $\sqrt{p}$ as $p \rightarrow \infty$.

Let $p<\infty$ be arbitrary. Denote

$$
Y_{n, p}=2^{\alpha n}\left\|G\left(\cdot+2^{-n}\right)-G\right\|_{L^{p}\left(I\left(2^{-n}\right) ; X\right)} .
$$

As in [15] we can write

$$
Y_{n, p}^{p}=\int_{0}^{1} 2^{-n} \sum_{m=1}^{2^{n}-1}\left\|\gamma_{n, m, s}\right\|^{p} d s
$$

where $\gamma_{n, m, s}=2^{\alpha n}\left(G\left((s+m) 2^{-n}\right)-G\left((s+m-1) 2^{-n}\right)\right)$ for $m=1, \ldots, 2^{n}-1$ and $n \geq 1$ and $s \in[0,1]$. Denote

$$
c_{p, n, m, s}^{p}=\mathbb{E}\left\|\gamma_{n, m, s}\right\|^{p} \leq c_{p}^{p}=\|G\|_{C^{\alpha}\left([0,1] ; L^{p}(\Omega ; X)\right)}^{p}
$$

and

$$
c_{p, n}^{p}=\int_{0}^{1} 2^{-n} \sum_{m=1}^{2^{n}-1} c_{p, n, m, s}^{p} d s .
$$

Then also $c_{p, n}^{p} \leq c_{p}^{p}$. 
It follows from Jensen's inequality that

$$
\begin{aligned}
a_{n} & :=\mathbb{E}\left(Y_{n, p}^{p}-c_{p, n}^{p}\right)^{2}=\mathbb{E}\left|\int_{0}^{1} 2^{-n} \sum_{m=1}^{2^{n}-1}\left[\left\|\gamma_{n, m, s}\right\|^{p}-c_{p, n, m, s}^{p}\right] d s\right|^{2} \\
& \leq \int_{0}^{1} \mathbb{E}\left|2^{-n} \sum_{m=1}^{2^{n}-1}\left[\left\|\gamma_{n, m, s}\right\|^{p}-c_{p, n, m, s}^{p}\right]\right|^{2} d s \\
& =\int_{0}^{1} 2^{-2 n} \sum_{k, m=1}^{2^{n}-1} \mathbb{E}\left(\left\|\gamma_{n, k, s}\right\|^{p}-c_{p, n, k, s}^{p}\right)\left(\left\|\gamma_{n, m, s}\right\|^{p}-c_{p, n, m, s}^{p}\right) d s .
\end{aligned}
$$

By Corollary 3.5 with $H=\mathbb{R}$ we obtain that for $m \neq k$,

$$
\begin{aligned}
& \mathbb{E}\left(\left\|\gamma_{n, k, s}\right\|^{p}-c_{p, n, k, s}^{p}\right)\left(\left\|\gamma_{n, m, s}\right\|^{p}-c_{p, n, m, s}^{p}\right) \\
& \quad \leq\left(K\left(2^{-n},|m-k| 2^{-n}\right)\right)^{2}\left(c_{2 p, n, k, s}^{2 p}-c_{p, n, k, s}^{2 p}\right)^{\frac{1}{2}}\left(c_{2 p, n, m, s}^{2 p}-c_{p, n, m, s}^{2 p}\right)^{\frac{1}{2}} \\
& \quad \leq\left(K\left(2^{-n},|m-k| 2^{-n}\right)\right)^{2} c_{2 p}^{2 p} .
\end{aligned}
$$

For $m=k$ we can write

$$
\mathbb{E}\left|\left\|\gamma_{n, k, s}\right\|^{p}-c_{p, n, k, s}^{p}\right|^{2}=c_{2 p, n, k, s}^{2 p}-c_{p, n, k, s}^{2 p} \leq c_{2 p, n, k, s}^{2 p}
$$

Therefore,

$$
\begin{aligned}
a_{n} & \leq 2^{-2 n} \sum_{k, m=1}^{2^{n}-1}\left(K\left(2^{-n},|m-k| 2^{-n}\right)\right)^{2} c_{2 p}^{2 p} \\
& \leq\left(2^{-n}+2^{-2 n} 2 \sum_{m=1}^{2^{n}-1} \sum_{k=1}^{m-1}\left(K\left(2^{-n},(m-k) 2^{-n}\right)\right)^{2}\right) c_{2 p}^{2 p} \\
& =\left(2^{-n}+2^{-2 n} 2 \sum_{m=1}^{2^{n}-1} \sum_{i=1}^{m-1}\left(K\left(2^{-n}, i 2^{-n}\right)\right)^{2}\right) c_{2 p}^{2 p} \\
& =C_{n} c_{2 p}^{2 p},
\end{aligned}
$$

where

$$
C_{n}=\left(2^{-n}+2^{-2 n} 2 \sum_{i=1}^{2^{n}-1}\left(2^{n}-i\right)\left(K\left(2^{-n}, i 2^{-n}\right)\right)^{2}\right) .
$$

By Eq. 5.2, $\sum_{n \geq 1} C_{n}<\infty$ holds, hence $\sum_{n} a_{n}<\infty$. From this we obtain that for all $\varepsilon>0$

$$
\sum_{n \geq 1} \mathbb{P}\left(\left|Y_{n, p}^{p}-c_{p, n}^{p}\right|>\varepsilon\right) \leq \varepsilon^{-2} \sum_{n \geq 1} a_{n}<\infty .
$$


Therefore the Borel-Cantelli lemma gives that

$$
\lim _{n \rightarrow \infty} Y_{n, p}^{p}-c_{p, n}^{p}=0 \text { a.s. }
$$

Since $c_{p, n} \leq c_{p}$ this implies that $Y_{n, p}$ is uniformly bounded a.s. This proves the first statement. Since Eq. 5.3 implies that $\lim _{\sup _{n \rightarrow \infty}} c_{p, n} \geq k_{p}$, the two final statement also follow.

We still need to show that $G \in B_{\Phi_{2}, \infty}^{\alpha}(0,1 ; X)$ a.s. We proved that $\mathbb{E}\left(Y_{n, p}^{p}-c_{p}^{p}\right)^{2} \leq$ $c_{2 p}^{2 p} C_{n}$. Therefore,

$$
\mathbb{E}\left(Y_{n, p}^{p} c_{p}^{-p}-1\right)^{2} \leq c_{2 p}^{2 p} c_{p}^{-2 p} C_{n} \leq K^{2 p} C_{n},
$$

where $K \geq 1$ is some constant. Hence for all $\lambda>1$,

$$
\mathbb{P}\left(Y_{n, p} c_{p}^{-1}>\lambda\right) \leq \mathbb{P}\left(\left|Y_{n, p}^{p} c_{p}^{-p}-1\right|>\lambda^{p}-1\right) \leq K^{2 p} C_{n}\left(\lambda^{p}-1\right)^{-2},
$$

and thus for $\lambda=2 K$

$$
\sum_{n, p=1}^{\infty} \mathbb{P}\left(Y_{n, p} c_{p}^{-1}>\lambda\right) \leq \sum_{n=1}^{\infty} C_{n} \sum_{p=1}^{\infty} K^{2 p}\left(\lambda^{p}-1\right)^{-2}<\infty
$$

so that by the Borel-Cantelli lemma

$$
\mathbb{P}\left(Y_{n, p} c_{p}^{-1}>\lambda \text { for infinitely many pairs }(n, p)\right)=0 .
$$

Since $c_{p} \approx p^{1 / 2}$ this means that a.s.

$$
\sup _{n, p} 2^{n \alpha}\left\|G\left(\cdot+2^{-n}\right)-G\right\|_{L^{p}\left(I\left(2^{-n}\right) ; X\right)} p^{-1 / 2}<\infty,
$$

hence $G \in B_{\Phi_{2}, \infty}^{\alpha}(0,1 ; X)$.

It would be interesting to construct a Gaussian process such that the assertion of Theorem 5.1 does not hold. Of course such a process does not satisfy Eqs. 5.1 and 5.2.

Problem 5.2 Let $\alpha \in(0,1)$ Does there exists a centered Gaussian process $G:[0,1] \times$ $\Omega \rightarrow \mathbb{R}$ such that $G \in C^{\alpha}\left([0,1] ; L^{2}(\Omega)\right)$ and $G \notin B_{p, \infty}^{\alpha}(0,1)$ a.s. for some $p<\infty$ ?

Next we apply Theorem 5.1 to fractional Brownian motions. Let $H \in(0,1)$. Recall that $G^{H}: \mathbb{R}_{+} \times \Omega \rightarrow \mathbb{R}$ is called an H-fractional Brownian $(H-f B M)$ if $G^{H}$ is a mean zero Gaussian process with $G^{H}(0)=0$ and

$$
\mathbb{E} G^{H}(t) G^{H}(s)=\frac{t^{2 H}+s^{2 H}-|t-s|^{2 H}}{2}
$$

for all $s, t \in \mathbb{R}_{+}$. A process $G^{H}: \mathbb{R}_{+} \times \Omega \rightarrow X$ is called an $H$-fractional Brownian motion $(H-f B M)$ if for all $x^{*} \in X^{*},\left\langle G^{H}, x^{*}\right\rangle$ is a constant times an $H$-fractional Brownian motion. One can check that $G^{H}$ is a stationary process and for each $t \in[0,1]$,

$$
G^{H}(t) \stackrel{D}{=} t^{H} G^{H}(1) .
$$


As a consequence of Theorem 5.1 we obtain the following vector valued extension of a result in [7]. The case $H=\frac{1}{2}$ has been considered in [15].

Corollary 5.3 Let $G^{H}: \mathbb{R}_{+} \times \Omega \rightarrow X$ be an nonzero $H$-fractional Brownian motion. Then for all $p \in[1, \infty), G^{H} \in B_{p, \infty}^{H}(0,1 ; X)$ a.s. and $G^{H} \in B_{\Phi_{2}, \infty}^{H}(0,1 ; X)$ a.s. Moreover, $\left\|G^{H}\right\|_{B_{p, \infty}^{H}(0,1 ; X)} \geq\left(\mathbb{E}\left\|G^{H}(1)\right\|^{p}\right)^{\frac{1}{p}}$ a.s. and for all $q \in[1, \infty), G^{H} \notin B_{p, q}^{H}(0,1 ; X)$ a.s.

Proof It follows from the above discussion that for all $s, t, a \in \mathbb{R}_{+}$and $x^{*}, y^{*} \in X^{*}$,

$$
\begin{aligned}
\mathbb{E}\left\langle G^{H}(t+a)-G^{H}(t), x^{*}\right\rangle & \left\langle G^{H}(s+a)-G^{H}(s), y^{*}\right\rangle \\
& =\left\langle Q x^{*}, y^{*}\right\rangle \frac{|t+a-s|^{2 H}+|t-a-s|^{2 H}-2|t-s|^{2 H}}{2} .
\end{aligned}
$$

Therefore,

$$
\rho\left(G^{H}(t+a)-G^{H}(t), G^{H}(s+a)-G^{H}(s)\right)=K(a,|t-s|) .
$$

where

$$
K(a,|t-s|)=\frac{|| t+a-\left.s\right|^{2 H}+|t-a-s|^{2 H}-2|t-s|^{2 H} \mid}{a^{2 H}} .
$$

It follows that for $a \in(0,1)$ and $b \in\left[1, a^{-1}\right]$,

$$
K(a, a b)=\left|(b+1)^{2 H}+(b-1)^{2 H}-2 b^{2 H}\right| .
$$

One easily checks that

$$
K(a, a b) \leq C_{H} b^{2 H-2}, a \in(0,1), b \in\left[1, a^{-1}\right],
$$

where $C$ only depends on $H$. Therefore, the condition of Theorem 5.1 (see the remarks below it) is satisfied, so Eq. 5.2 is fulfilled.

By stationarity and Eq. 5.4 we obtain that for $t>s$

$$
\mathbb{E}\left\|G^{H}(t)-G^{H}(s)\right\|^{p}=\mathbb{E}\left\|G^{H}(t-s)\right\|^{p}=(t-s)^{H p} \mathbb{E}\left\|G^{H}(1)\right\|^{p} .
$$

The first result now follows from Theorem 5.1. The estimate $\left\|G^{H}\right\|_{B_{p, \infty}^{H}(0,1 ; X)} \geq$ $\left(\mathbb{E}\left\|G^{H}(1)\right\|^{p}\right)^{\frac{1}{p}}$ follows from Theorem 5.1, since by Eq. 5.4

$$
\mathbb{E}\left\|G^{H}(t)\right\|^{p}=t^{H p} \mathbb{E}\left\|G^{H}(1)\right\|^{p} .
$$

Next we consider the case that $G:[0,1] \times \Omega \rightarrow X$ is of the form

$$
G(t)=\sum_{n \geq 1} G_{n}(t) x_{n}
$$

Here $\left(G_{n}\right)_{n \geq 1}$ are centered real-valued independent Gaussian processes on $[0,1]$ with

$$
\sup _{n \geq 1} \sup _{t \in[0,1]} \mathbb{E}\left|G_{n}(t)\right|^{2}<\infty,
$$


and $\left(x_{n}\right)_{n \geq 1}$ in $X$ are such that $\sum_{n \geq 1} \gamma_{n} x_{n}$ converges in $L^{2}(\Omega ; X)$ where $\left(\gamma_{n}\right)_{n \geq 1}$ is a sequence of independent standard Gaussian random variables. By the Kahane contraction principle for each $t \in[0,1], G(t)$ is well-defined in $L^{2}(\Omega ; X)$. As it turns out, in this situation it suffices to assume Eq. 5.1 for each of the processes $G_{n}$.

Corollary 5.4 Let $G$ be as above. Let $\alpha \in(0,1)$. Assume that for all $n \geq 1$ and for all $s, t, a, b \in[0,1]$ with $s+a, t+a \in[0,1]$ and $(s, s+a) \cap(t, t+a)=\emptyset$ it holds that

$$
\rho\left(G_{n}(t+a)-G_{n}(t), G_{n}(s+a)-G_{n}(s)\right) \leq K(a,|t-s|),
$$

with $K:[0,1]^{2} \rightarrow[0,1]$ such that Eq. 5.2 holds. If for some $p \in[1, \infty), G \in$ $C^{\alpha}\left([0,1] ; L^{p}(\Omega ; X)\right)$ then for all $p \in[1, \infty), G \in B_{p, \infty}^{\alpha}(0,1 ; X)$ a.s. Moreover, $G \in$ $B_{\Phi_{2}, \infty}^{\alpha}(0,1 ; X)$ a.s.

Proof We only need to show Eq. 5.1 for $G$. Let us denote $\Delta G_{n}(t, a)=G_{n}(t+a)-$ $G_{n}(t)$. For $x^{*}, y^{*} \in X^{*}$ we can estimate

$$
\begin{aligned}
& \left|\mathbb{E}\left\langle G(t+a)-G(t), x^{*}\right\rangle\left\langle G(s+a)-G(s), y^{*}\right\rangle\right| \\
& \quad=\left|\sum_{n \geq 1} \mathbb{E}\left(\Delta G_{n}(t, a) \Delta G_{n}(s, a)\right)\left\langle x_{n}, x^{*}\right\rangle\left\langle x_{n}, y^{*}\right\rangle\right| \\
& \quad \leq \sum_{n \geq 1}\left|\mathbb{E}\left(\Delta G_{n}(t, a) \Delta G_{n}(s, a)\right)\right|\left|\left\langle x_{n}, x^{*}\right\rangle\right|\left|\left\langle x_{n}, y^{*}\right\rangle\right| \\
& \quad \leq K(a,|t-s|) \sum_{n \geq 1}\left(\mathbb{E}\left|\Delta G_{n}(t, a)\right|^{2}\right)^{\frac{1}{2}}\left(\mathbb{E}\left|\Delta G_{n}(s, a)\right|^{2}\right)^{\frac{1}{2}}\left|\left\langle x_{n}, x^{*}\right\rangle\right|\left|\left\langle x_{n}, y^{*}\right\rangle\right| \\
& \quad \leq K(a,|t-s|)\left(\sum_{n \geq 1} \mathbb{E}\left|\Delta G_{n}(t, a)\right|^{2}\left|\left\langle x_{n}, x^{*}\right\rangle\right|^{2}\right)^{\frac{1}{2}}\left(\sum_{n \geq 1} \mathbb{E}\left|\Delta G_{n}(s, a)\right|^{2}\left|\left\langle x_{n}, y^{*}\right\rangle\right|^{2}\right)^{\frac{1}{2}} \\
& \quad=K(a,|t-s|)\left(\mathbb{E}\left\langle G(t+a)-G(t), x^{*}\right\rangle^{2}\right)^{\frac{1}{2}}\left(\mathbb{E}\left\langle G(s+a)-G(s), y^{*}\right\rangle^{2}\right)^{\frac{1}{2}} .
\end{aligned}
$$

The result follows from this.

Remark 5.5 In Eq. 5.6 and Corollary 5.4 the assumption that $\left(G_{n}\right)_{n \geq 1}$ are independent is not needed. It suffices to assume the correlation behaves properly. Let $\rho_{i j}(t, s, a)=\rho\left(\Delta G_{i}(t, a), \Delta G_{j}(s, a)\right)$. Assume that (compare Eq. 4.1)

$$
C:=\sup \sup _{i \geq 1} \sum_{j \geq 1}\left|\rho_{i j}(t, s, a)\right|,
$$

where the supremum is taken over all $t, s \in[0,1)$ and $a \in[0,1]$ such that $t+a, s+a \in$ $[0,1]$. Then by Proposition $4.10, G$ is well-defined. If each $G_{n}$ satisfies the conditions (5.7) and $K$ satisfies Eq. 5.2, then using similar arguments as in the proofs of Lemma 4.8 and Proposition 4.10 one can show that Eq. 5.1 holds and therefore the assertion in Corollary 5.4 extends to the case of Gaussian processes $\left(G_{n}\right)_{n \geq 1}$ which are not necessarily independent and satisfy Eq. 5.8. 
Another application of Theorem 5.1 is the following.

Corollary 5.6 Let $r \in(2, \infty]$ and $\phi \in L^{r}(0,1)$. Let $(W(t))_{t \in[0,1]}$ be a standard Brownian motion. Then the integral process $\zeta(t)=\int_{0}^{t} \phi d W$ satisfies $\zeta \in B_{p, \infty}^{\frac{1}{2}-\frac{1}{r}}(0,1)$ a.s. for all $p \in[1, \infty)$ and $\zeta \in B_{\Phi_{2}, \infty}^{\frac{1}{2}-\frac{1}{r}}(0,1)$ a.s.

This result can be generalized in various ways. It has an extension to the case where $\phi$ takes its values in a Banach space $X$ with type 2. Further, one may consider integration with respect to other Gaussian processes for which a stochastic integration theory exists.

For $r=\infty$, in [27] Roynette considers the more general situation that $\phi$ is an adapted process with $\phi \in L^{\infty}(0,1)$ a.s.

Proof Since $\zeta$ has independent increments, it suffices to show that $\zeta$ is $\left(\frac{1}{2}-\frac{1}{r}\right)$ Hölder continuous in second moment. Let $0 \leq s_{1}<s_{2} \leq 1$. Then using the $L^{2}$ isometric property of the stochastic integral and Hölder's inequality, we deduce

$$
\left(\mathbb{E}\left|\zeta\left(s_{2}\right)-\zeta\left(s_{1}\right)\right|^{2}\right)^{\frac{1}{2}}=\left(\int_{s_{1}}^{s_{2}} \phi^{2}(t) d t\right)^{\frac{1}{2}} \leq\left(s_{2}-s_{1}\right)^{\frac{1}{2}-\frac{1}{r}}\|\phi\|_{L^{r}(0,1)} .
$$

If $G \in B_{p, \infty}^{\alpha}(0,1 ; X)$ a.s., then as in [15] one can show that $G: \Omega \rightarrow B_{p, \infty}^{\alpha}(0,1 ; X)$ is a centered Gaussian in a slightly different sense than we defined before. Namely it does not have to be a measurable mapping in general and it does not have to be Radon. Let us recall some definitions (cf. [20, Section 3.1]).

Let $X$ be a Banach space with the property that there exists a norming sequence of functionals, i.e. there exist $x_{1}^{*}, x_{2}^{*}, \ldots$ in $B_{X^{*}}$ such that for all $x \in X,\|x\|=$ $\sup _{n \geq 1}\left|\left\langle x, x_{n}^{*}\right\rangle\right|$. Examples of such Banach spaces are all separable Banach spaces, but also spaces like $l^{\infty}$ and $B_{p, \infty}^{\alpha}(0,1 ; Y)$ for a separable Banach space $Y$.

Let $X$ be a Banach space with norming sequence $\left(x_{n}^{*}\right)_{n \geq 1}$. A mapping $\xi: \Omega \rightarrow X$ will be called a centered Gaussian if for all $x^{*} \in \operatorname{span}\left\{x_{n}^{*}: n \geq 1\right\}$ the random variable $\left\langle\xi, x^{*}\right\rangle$ is a centered Gaussian. For a centered Gaussian random variable we define

$$
\sigma(\xi)=\sup _{n \geq 1}\left(\mathbb{E}\left|\left\langle\xi, x_{n}^{*}\right\rangle\right|^{2}\right)^{1 / 2}
$$

The value of $\sigma$ is independent of the norming sequence $\left(x_{n}^{*}\right)_{n \geq 1}$ (cf. [20, Section 3.1]). Note that we do not assume that $\xi$ is strongly measurable. However, by the assumptions $\|\xi\|$ is always measurable.

Let us turn back to the mapping $G: \Omega \rightarrow B_{p, \infty}^{\alpha}(0,1 ; X)$ that one can consider in the setting of Theorem 5.1. As in [15] one can show that $G$ is a centered Gaussian. The centered Gaussian $G: \Omega \rightarrow B_{p, \infty}^{\alpha}(0,1 ; X)$ has the peculiar property that $\|G\|_{B_{p, \infty}^{\alpha}(0,1 ; X)} \geq k_{p}$ a.s. Therefore, it is not Radon (cf. [20, Section 3.1]).

Due to the fact that $G$ is a centered Gaussian Fernique's theorem implies that there exists an $\varepsilon>0$ such that $\mathbb{E} \exp \left(\varepsilon\|G\|_{B_{p, \infty}^{\alpha}(0,1 ; X)}^{2}\right)<\infty$. Using Lemma 5.9 and [20, Corollary 3.2] one can even show that $\varepsilon>0$ can be chosen independent of $p \in[1, \infty)$. Similarly, there exists an $\varepsilon>0$ such that $\mathbb{E} \exp \left(\varepsilon\|G\|_{B_{\Phi_{2}, \infty}^{\alpha}(0,1 ; X)}^{2}\right)<\infty$. 
In the next result we will estimate $\mathbb{E}\|G\|_{B_{p, \infty}^{\alpha}(0,1 ; X)}$ and $\mathbb{E}\|G\|_{B_{\Phi_{2}, \infty}^{\alpha}(0,1 ; X)}$. This extends [15, Theorem 6.1] where the case that $G$ is a vector valued Brownian has been considered. The assumption on the correlation structure will be slightly stronger than the assumption in Theorem 5.1. It is the same condition as below Theorem 5.1. There exists an $\varepsilon>0$ such that

$$
K(a, a b) \leq C b^{-\varepsilon}, \quad a \in(0,1), \quad b \in\left[1, a^{-1}\right] .
$$

Theorem 5.7 Let $X$ be a Banach space. Let $\alpha \in(0,1)$. Let $\left(G_{t}\right)_{t \in[0,1]}$ be an $X$ valued centered Gaussian process starting at zero with the property that for all $s, t, a, b \in[0,1]$ with $s+a, t+a \in[0,1]$ and $(s, s+a) \cap(t, t+a)=\emptyset$, Eq. 5.1 holds where $K:[0,1]^{2} \rightarrow[0,1]$ satisfies Eq. 5.10. Assume that for some $p \in[1, \infty), G \in$ $C^{\alpha}\left([0,1] ; L^{p}(\Omega ; X)\right)$. Then for all $p \in[1, \infty)$,

$$
\begin{aligned}
& \mathbb{E}\|G\|_{B_{p, \infty}^{\alpha}(0,1 ; X)} \lesssim K\|G\|_{\alpha, p}, \\
& \mathbb{E}\|G\|_{B_{\Phi_{2}, \infty}^{\alpha}(0,1 ; X)} \lesssim K\|G\|_{\alpha, 1} .
\end{aligned}
$$

Here

$$
\|G\|_{\alpha, p}=\sup _{s \neq t} \frac{\left(\mathbb{E}\|G(t)-G(s)\|^{p}\right)^{\frac{1}{p}}}{|t-s|^{\alpha}} .
$$

The inequalities (5.11) and (5.12) are new even in the case $X=\mathbb{R}$. The constant in the inequality depends on $\varepsilon$ and contains a factor $(C+1)^{\frac{1}{2}}$, where $\varepsilon$ and $C$ are as in Eq. 5.10 .

Notice that if Eq. 5.3 holds for some $p \in[1, \infty)$, then Theorem 5.1 also yields the following estimates from below hold:

$$
\begin{aligned}
\mathbb{E}\|G\|_{B_{p, \infty}^{\alpha}(0,1 ; X)} \geq k_{p}, \\
\mathbb{E}\|G\|_{B_{\Phi_{2}, \infty}^{\alpha}(0,1 ; X)} \geq p^{-\frac{1}{2}} k_{p} .
\end{aligned}
$$

Recall that for $H$-fBMs we already obtained Eq. 5.10 in Eq. 5.5. Therefore, as a consequence of Theorem 5.7 we have the following result.

Corollary 5.8 Let $X$ be a Banach space. Let $p \in[1, \infty)$. Let $H \in(0,1)$. For an $X$-valued $H$-fractional Brownian motion $G^{H}$ we have

$$
\begin{gathered}
\mathbb{E}\left\|G^{H}\right\|_{B_{p, \infty}^{H}(0,1 ; X)} \bar{\sim}_{H}\left(\mathbb{E}\left\|G^{H}(1)\right\|^{p}\right)^{1 / p}, \\
\mathbb{E}\left\|G^{H}\right\|_{B_{\Phi_{2}, \infty}^{H}(0,1 ; X)} \bar{\sim}_{H} \mathbb{E}\left\|G^{H}(1)\right\| .
\end{gathered}
$$

For a centered Gaussian Radon random variable $\xi$ with values in a Banach space $X$, let $Q_{\xi} \in \mathcal{B}\left(X^{*}, X\right)$ denote its covariance operator, i.e.

$$
Q_{\xi} x^{*}=\mathbb{E}\left\langle\xi, x^{*}\right\rangle \xi .
$$

The following estimate for $Q_{\xi}$ will be needed: for all $p \in[1, \infty)$, we have

$$
\left\|Q_{\xi}\right\|^{\frac{1}{2}} \lesssim p^{-1 / 2}\left(\mathbb{E}\|\xi\|^{p}\right)^{\frac{1}{p}}
$$


Indeed,

$$
\left\|Q_{\xi}\right\|^{\frac{1}{2}}=\sup _{x^{*} \in B_{X^{*}}}\left(\mathbb{E}\left|\left\langle\xi, x^{*}\right\rangle\right|^{2}\right)^{\frac{1}{2}} \lesssim p^{-\frac{1}{2}} \sup _{x^{*} \in B_{X^{*}}}\left(\mathbb{E}\left|\left\langle\xi, x^{*}\right\rangle\right|^{p}\right)^{\frac{1}{p}} \leq p^{-\frac{1}{2}}\left(\mathbb{E}\|\xi\|^{p}\right)^{\frac{1}{p}}
$$

For the proof of Theorem 5.7 we need the following lemmas.

Lemma 5.9 Let $\left(G_{t}\right)_{t \in[0,1]}$ be an $X$-valued centered Gaussian process with the property that for all $s, t, a, b \in[0,1]$ with $s+a, t+a \in[0,1]$ and $(s, s+a) \cap(t, t+a)=\emptyset$, Eq. 5.1 holds where $K:[0,1]^{2} \rightarrow[0,1]$ satisfies Eq. 5.10. Let $c \in(0,1)$. Let $p \in$ $[1, \infty)$. Consider $\Delta G(\cdot, c)=G(\cdot+c)-G$ as an $L^{p}(0,1-c, X)$-valued Gaussian random variable. Then

$$
\sigma(\Delta G(\cdot, c)) \lesssim_{K} \sup _{t \in[0,1]}\left\|Q_{\Delta G(t, c)}\right\|^{\frac{1}{2}} c^{1 / p}
$$

The lemma is similar as [15, Lemma 6.2] where the case $G$ is a vector valued Brownian motion is considered. The proof in [15] uses stochastic integration theory. Since we cannot use a "good" stochastic integration theory for our class of Gaussian processes, we give a different argument.

Proof We may assume $\varepsilon \in(0,1)$. Secondly we may assume $p>\frac{2}{\varepsilon}$. Indeed, if the lemma holds for $p \in\left(\frac{2}{\varepsilon}, \infty\right)$, then let $q=\frac{2}{\varepsilon}+1$. For $p \in\left[1, \frac{2}{\varepsilon}\right)$ it follows from $\|\phi\|_{L^{p}(0,1-c ; X)} \leq\|\phi\|_{L^{q}(0,1-c ; X)}$ and $c^{\frac{1}{q}} \leq c^{\frac{1}{p}}$ that

$$
\begin{aligned}
\sigma_{L^{p}(0,1-c ; X)}(G(\cdot+c)-G) & \leq \sigma_{L^{q}(0,1-c ; X)}(G(\cdot+c)-G) \\
& \lesssim_{K} \sup _{t \in[0,1]}\left\|Q_{\Delta G(t, c)}\right\|^{\frac{1}{2}} c^{1 / p} .
\end{aligned}
$$

Fix $p \in\left(\frac{2}{\varepsilon}, \infty\right)$ and $f \in L^{p^{\prime}}\left(0,1-c ; X^{*}\right)$. In particular note that $p>2$. We use the same symbol for the extension of $f$ to $\mathbb{R}$ as being zero outside $(0,1-c)$. By Fubini's theorem and symmetry we can write

$$
\begin{aligned}
\mathbb{E}\left|\int_{0}^{1-c}\langle\Delta G(t, c), f(t)\rangle d t\right|^{2} & =\int_{0}^{1-c} \int_{0}^{1-c} \mathbb{E}\langle\Delta G(t, c), f(t)\rangle\langle\Delta G(s, c), f(s)\rangle d s d t \\
& =2 \int_{0}^{1-c} \int_{0}^{t} \mathbb{E}\langle\Delta G(t, c), f(t)\rangle\langle\Delta G(s, c), f(s)\rangle d s d t
\end{aligned}
$$

By the assumptions (5.1) and (5.10) for all $s \in(0, t-c)$, we have

$$
\begin{aligned}
\mathbb{E}\langle\Delta G(t, c), f(t)\rangle\langle\Delta G(s, c), f(s)\rangle & \leq\left\|Q_{\Delta G(\cdot, c)}\right\|\|f(t)\|\|f(s)\| K(c, t-s) \\
& \leq C\left\|Q_{\Delta G(t, c)}\right\|^{\frac{1}{2}}\left\|Q_{\Delta G(s, c)}\right\|^{\frac{1}{2}}\|f(t)\|\|f(s)\| c^{\varepsilon}(t-s)^{-\varepsilon} .
\end{aligned}
$$


Let $g:(-\infty,-c) \rightarrow \mathbb{R}$ be defined as $g(s)=(-s)^{-\varepsilon}$. By Hölder's inequality, Fubini's theorem and Young's inequality we obtain that

$$
\begin{aligned}
\int_{0}^{1-c} & \int_{0}^{t-c} \mathbb{E}\langle\Delta G(t, c), f(t)\rangle\langle\Delta G(s, c), f(s)\rangle d s d t \\
& \leq C \sup _{t \in[0,1]}\left\|Q_{\Delta G(t, c)}\right\| c^{\varepsilon} \int_{\mathbb{R}} \int_{\mathbb{R}}\|f(t)\|\|f(s)\| \mathbf{1}_{(0, t-c)}(s)(t-s)^{-\varepsilon} d s d t \\
& =C \sup _{t \in[0,1]}\left\|Q_{\Delta G(t, c)}\right\| c^{\varepsilon} \int_{\mathbb{R}}\left(\left(\mathbf{1}_{(-\infty,-c)} g\right) *\|f\|\right)(s)\|f(s)\| d s \\
& \leq C \sup _{t \in[0,1]}\left\|Q_{\Delta G(t, c)}\right\| c^{\varepsilon}\|f\|_{L^{p^{\prime}}\left(0,1-c ; X^{*}\right)}\left\|\left(\mathbf{1}_{(-\infty,-c)} g\right) *\right\| f \|_{L^{p}(\mathbb{R})} \\
& \leq C \sup _{t \in[0,1]}\left\|Q_{\Delta G(t, c)}\right\| c^{\varepsilon}\|f\|_{L^{p^{\prime}}\left(0,1-c ; X^{*}\right)}^{2}\left\|\mathbf{1}_{(-\infty,-c)} g\right\|_{L^{\frac{p}{2}}(\mathbb{R})} \\
& \lesssim C \sup _{t \in[0,1]}\left\|Q_{\Delta G(t, c)}\right\|\|f\|_{L^{p^{\prime}}\left(0,1-c ; X^{*}\right)}^{2} c^{\frac{2}{p}}
\end{aligned}
$$

where in the last line we used $p>\frac{2}{\varepsilon}$.

For the other part again by Hölder's inequality, Fubini's theorem and Young's inequality,

$$
\begin{aligned}
\int_{0}^{1-c} & \int_{t-c}^{t} \mathbb{E}\langle\Delta G(t, c), f(t)\rangle\langle\Delta G(s, c), f(s)\rangle d s d t \\
& \leq \sup _{t \in[0,1]}\left\|Q_{\Delta G(t, c)}\right\| \int_{0}^{1-c} \int_{t-c}^{t}\|f(t)\|\|f(s)\| d s d t \\
& \leq \sup _{t \in[0,1]}\left\|Q_{\Delta G(t, c)}\right\| \int_{\mathbb{R}}\left(\mathbf{1}_{(-c, 0)} *\|f\|\right)(s)\|f(s)\| d s \\
& \leq \sup _{t \in[0,1]}\left\|Q_{\Delta G(t, c)}\right\|\|f\|_{L^{p^{\prime}\left(0,1-c ; X^{*}\right)}}\left\|\mathbf{1}_{(-c, 0)} *\right\| f\|\|_{L^{p}(\mathbb{R})} \\
& \leq \sup _{t \in[0,1]}\left\|Q_{\Delta G(t, c)}\right\|\|f\|_{L^{p^{\prime}\left(0,1-c ; X^{*}\right)}}^{2}\left\|\mathbf{1}_{(-c, 0)}\right\|_{L^{\frac{p}{2}}(\mathbb{R})} \\
& =\sup _{t \in[0,1]}\left\|Q_{\Delta G(t, c)}\right\|\|f\|_{L^{p^{\prime}\left(0,1-c ; X^{*}\right)}}^{2} c^{\frac{2}{p}} .
\end{aligned}
$$

Therefore, we can conclude that

$$
\begin{aligned}
\left(\mathbb{E} \mid \int_{0}^{1-c}\right. & \left.\left.\langle\Delta G(t, c), f(t)\rangle d t\right|^{2}\right)^{1 / 2} \\
& \lesssim_{\varepsilon}(C+1)^{\frac{1}{2}} \sup _{t \in[0,1]}\left\|Q_{\Delta G(t, c)}\right\|^{\frac{1}{2}}\|f\|_{L^{p^{\prime}}\left(0,1-c ; X^{*}\right)} c^{\frac{1}{p}}
\end{aligned}
$$

Since, $L^{p^{\prime}}\left(0,1-c ; X^{*}\right)$ is norming for $L^{p}(0,1-c ; X)$ the result follows.

Corollary 5.10 Let $\left(G_{t}\right)_{t \in[0,1]}$ be an $X$-valued centered Gaussian process with the property that for all $s, t, a, b \in[0,1]$ with $s+a, t+a \in[0,1]$ and $(s, s+a) \cap(t, t+a)=\emptyset$, 
Eq. 5.1 holds where $K:[0,1]^{2} \rightarrow[0,1]$ satisfies Eq. 5.10. Let $c \in\left(0, e^{-1 / 2}\right]$. Consider $G(\cdot+c)-G$ as an $\mathfrak{L}^{\Phi_{2}}(0,1-c ; X)$-valued Gaussian random variable. Then

$$
\sigma(G(\cdot+c)-G) \lesssim_{K} \sup _{t \in[0,1]}\left\|Q_{\Delta G(t, c)}\right\|^{\frac{1}{2}}\left(\log c^{-1}\right)^{-1 / 2} .
$$

This follows from Lemma 5.9 using similar arguments as in the proof of [15, Corollary 6.1].

We are now ready to prove Theorem 5.7. It is an extension of [15, Theorem 6.1].

Proof of Theorem 5.7 Since $G(0)=0$, the $L^{p}$-part of the norm in $B_{p, \infty}^{\alpha}(0,1 ; X)$ can be estimated as

$$
\begin{aligned}
\mathbb{E}\|G\|_{L^{p}(0,1 ; X)} & \leq\left(\mathbb{E}\|G\|_{L^{p}(0,1 ; X)}^{p}\right)^{\frac{1}{p}}=\left(\int_{0}^{1} \mathbb{E}\|G(t)-G(0)\|^{p} d t\right)^{\frac{1}{p}} \\
& \leq\left(\int_{0}^{1} t^{\alpha p}\|G\|_{\alpha, p}^{p} d t\right)^{\frac{1}{p}} \leq\|G\|_{\alpha, p} .
\end{aligned}
$$

For the other part of the Besov norm we consider

$$
\mathbb{E} \sup _{n \geq 1} 2^{n \alpha}\left\|G\left(\cdot+2^{-n}\right)-G\right\|_{L^{p}\left(0,1-2^{-n} ; X\right)}
$$

To estimate this, we apply Theorem 4.4 to the $L^{p}(0,1 ; X)$-valued Gaussian random variables $\xi_{n}=2^{n \alpha}\left[G\left(\cdot+2^{-n}\right)-G\right] 1_{\left[0,1-2^{-n}\right]}$ :

$$
\mathbb{E} \sup _{n \geq 1}\left\|\xi_{n}\right\| \lesssim \sup _{n \geq 1} \mathbb{E}\left\|\xi_{n}\right\|+\left\|\left(\sigma_{n}\right)_{n \geq 1}\right\|_{\Theta}
$$

where $\sigma_{n}$ are the weak variances of $\xi_{n}$ in $L^{p}(0,1 ; X)$.

The first term is smaller than $\|G\|_{\alpha, p}$. For the second term note that by Eq. 5.16,

$$
\left\|Q_{\xi_{n}}\right\|^{\frac{1}{2}} \lesssim p^{-\frac{1}{2}}\|G\|_{\alpha, p}
$$

By Eq. 5.18 and Lemma 5.9 we the Hölder continuity assumption we obtain

$$
\begin{aligned}
\sigma_{n}=2^{n \alpha} \sigma_{L^{p}\left(0,1-2^{-n} ; X\right)}\left(\Delta G\left(t, 2^{-n}\right)\right) & \lesssim_{K} 2^{n \alpha} \sup _{t \in[0,1]}\left\|Q_{\Delta G\left(t, 2^{-n}\right)}\right\|^{\frac{1}{2}} 2^{-\frac{n}{p}} \\
& =\sup _{t \in[0,1]}\left\|Q_{\xi_{n}}\right\|^{\frac{1}{2}} 2^{-\frac{n}{p}} \lesssim p^{-\frac{1}{2}}\|G\|_{\alpha, p} 2^{-\frac{n}{p}}
\end{aligned}
$$

Therefore, using [15, Example 2.1] we obtain

$$
\begin{aligned}
\left\|\left(\sigma_{n}\right)_{n \geq 1}\right\|_{\Theta} & \lesssim_{K} p^{-\frac{1}{2}}\|G\|_{\alpha, p}\left\|\left(2^{-n / p}\right)_{n \geq 1}\right\|_{\Theta} \\
& \approx p^{-\frac{1}{2}}\|G\|_{\alpha, p} \sqrt{\log \left(1-2^{-1 / p}\right)^{-1}} \\
& \approx\|G\|_{\alpha, p} p^{-\frac{1}{2}}(1+\sqrt{\log p}) .
\end{aligned}
$$

Since $p^{-\frac{1}{2}}(1+\sqrt{\log p})$ is uniformly bounded for $p \in[1, \infty)$, the proof of Eq. 5.11 is complete. 
Next, we show Eq. 5.12. By [15, Equation (2.2)] we can write

$$
\begin{aligned}
& \mathbb{E}\|G\|_{B_{\Phi_{2}, \infty}^{\alpha}(0,1 ; X)} \\
& \quad \leq \mathbb{E}\|G\|_{L^{\Phi_{2}(0,1 ; X)}}+\mathbb{E} \sup _{n \geq 1} 2^{n \alpha}\left\|G\left(\cdot+2^{-n}\right)-G\right\|_{\mathcal{L}^{\Phi_{2}}\left(0,1-2^{-n} ; X\right)} .
\end{aligned}
$$

The first term can again be estimated as

$$
\begin{aligned}
\mathbb{E}\|G\|_{L^{\Phi_{2}(0,1 ; X)}} & \leq \mathbb{E} \inf _{\lambda>0} \frac{1}{\lambda} \int_{0}^{1} \mathbb{E} \exp \left(\lambda^{2}\|G(t)\|^{2}\right) d t \\
& \leq \inf _{\lambda>0} \frac{1}{\lambda} \int_{0}^{1} \mathbb{E} \exp \left(\lambda^{2}\|G(t)\|^{2}\right) d t .
\end{aligned}
$$

By a power series expansion and the Kahane-Khinchine inequalities (cf. [20, Corollary 3.2]) we obtain that

$$
\begin{aligned}
\mathbb{E} \exp \left(\lambda^{2}\|G(t)\|^{2}\right) d t & =\sum_{k \geq 0} \frac{\lambda^{2 k}}{k !} \mathbb{E}\|G(t)\|^{2 k} \\
& \leq\left[1+\sum_{k \geq 1} \frac{\lambda^{2 k}}{k !}(L \sqrt{2 k} \mathbb{E}\|G(t)\|)^{2 k}\right] \\
& \leq\left[1+\sum_{k \geq 1}\left(2 e[\lambda L \mathbb{E}\|G(t)\|]^{2}\right)^{k}\right], \\
& \leq\left[1+\sum_{k \geq 1}\left(2 e\left[\lambda L\|G\|_{\alpha, 1}\right]^{2}\right)^{k}\right],
\end{aligned}
$$

where $L$ is an absolute constant from the Gaussian norm comparison result [20, Corollary 3.2], and we used $k^{k} / k ! \leq e^{k}$. Letting $\lambda=\left(2 e L\|G\|_{\alpha, 1}\right)^{-1}$, we find that

$$
\mathbb{E}\|G\|_{L^{\Phi_{2}(0,1 ; X)}} \lesssim\|G\|_{\alpha, 1} .
$$

The second term in Eq. 5.19 can be estimated as before using Theorem 4.4. Consider the $\mathfrak{L}^{\Phi_{2}}(0,1 ; X)$-valued Gaussian random variables $\xi_{n}=2^{n / 2}\left[G\left(\cdot+2^{-j}\right)-\right.$ $G] 1_{\left[0,1-2^{-n}\right]}$. Let $\sigma_{n}$ denote the weak variance of $\xi_{n}$. In the same way as in Eq. 5.20 one can show

$$
\mathbb{E}\left\|\xi_{n}\right\|_{\mathfrak{L}^{\Phi_{2}(0,1 ; X)}} \leq \mathbb{E}\left\|\xi_{n}\right\|_{L^{\Phi_{2}(0,1 ; X)}} \lesssim\|G\|_{\alpha, 1} .
$$

By Theorem 4.4, [15, Remark 3.1], Corollary 5.10 and Eq. 5.18, it follows that

$$
\begin{aligned}
\mathbb{E} \sup _{n \geq 1}\left\|\xi_{n}\right\|_{\mathfrak{L}^{\Phi_{2}(0,1 ; X)}} & \lesssim \sup _{n \geq 1} \mathbb{E}\left\|\xi_{n}\right\|_{\mathcal{L}^{\Phi}(0,1 ; X)}+\left(\sum_{n \geq 1} \sigma_{n}^{4}\right)^{1 / 4}, \\
& \lesssim K\|G\|_{\alpha, 1}+\|G\|_{\alpha, 1}\left(\sum_{n \geq 1} n^{-2}\right)^{\frac{1}{4}} .
\end{aligned}
$$

This clearly gives the result. 
Acknowledgements The author thanks Jan Maas and Jan van Neerven for helpful comments. In particular, Jan Maas brought [23, Lemma 1.4.1] and [21, Lemma 6.4] to our attention. The author also thanks the anonymous referee for carefully reading the manuscript.

Open Access This article is distributed under the terms of the Creative Commons Attribution Noncommercial License which permits any noncommercial use, distribution, and reproduction in any medium, provided the original author(s) and source are credited.

\section{References}

1. Beśka, M., Ciesielski, Z.: Ergodic type theorems for Gaussian systems. Tr. Mat. Inst. Steklova 248 (2005), no. Issled. po Teor. Funkts. i Differ. Uravn., 40-45

2. Beśka, M., Ciesielski, Z.: Gebelein's inequality and its consequences. In: Approximarion and Probability, Banach Center Publ., vol. 72, pp. 11-23. Polish Academy of Sciences, Warsaw (2006)

3. Bogachev, V.I.: Gaussian measures, Mathematical Surveys and Monographs, vol. 62. American Mathematical Society, Providence (1998)

4. Burkholder, D.L.: Martingales and Singular Integrals in Banach Spaces. Handbook of the Geometry of Banach Spaces, vol. I, pp. 233-269. North-Holland, Amsterdam (2001)

5. Ciesielski, Z.: Modulus of smoothness of the Brownian paths in the $L^{p}$ norm. In: Constructive theory of functions (Varna, Bulgaria, 1991), pp. 71-75. Bulgarian Academy of Sciences, Varna (1991)

6. Ciesielski, Z.: Orlicz spaces, spline systems, and Brownian motion. Constr. Approx. 9(2-3), 191-208 (1993)

7. Ciesielski, Z., Kerkyacharian, G., Roynette, B.: Quelques espaces fonctionnels associés à des processus gaussiens. Stud. Math. 107(2), 171-204 (1993)

8. Davies, E.B., Simon, B.: $L^{1}$-properties of intrinsic Schrödinger semigroups. J. Funct. Anal. 65(1), 126-146 (1986)

9. Dym, H., McKean, H.P.: Gaussian Processes, Function Theory, and the Inverse Spectral Problem. Probability and Mathematical Statistics, vol. 31. Academic [Harcourt Brace Jovanovich], New York (1976)

10. Etemadi, N.: An elementary proof of the strong law of large numbers. Z. Wahrsch. Verw. Gebiete 55(1), 119-122 (1981)

11. Etemadi, N.: On the laws of large numbers for nonnegative random variables. J. Multivar. Anal. 13(1), 187-193 (1983)

12. Feyel, D., de La Pradelle, A.: On fractional Brownian processes. Potential Anal. 10(3), 273-288 (1999)

13. Gebelein, H.: Das statistische Problem der korrelation als variations- und eigenwertproblem und sein zusammenhang mit der ausgleichsrechnung. Z. Angew. Math. Mech. 21, 364-379 (1941)

14. Hoffmann-Jørgensen, J.: Probability in Banach space. In: École d'Été de Probabilités de Saint-Flour, VI -1976. Lecture Notes in Math, vol. 598, pp. 1-186. Springer, Berlin (1977)

15. Hytönen, T.P., Veraar, M.C.: On Besov regularity of Brownian motions in infinite dimensions. Probab. Math. Stat. 28(1), 143-162 (2008)

16. Kolmogorov, A.N., Rozanov, Ju.A.: On a strong mixing condition for stationary Gaussian processes. Teor. Veroyatnost. i Primenen. 5, 222-227 (1960)

17. König, H.: Eigenvalue Distribution of Compact Operators, Operator Theory: Advances and Applications, vol. 16. Birkhäuser, Basel (1986)

18. Krasnosel'skiı̌, M.A., Rutickiı̆, Ja.B.: Convex functions and Orlicz spaces. Translated from the first Russian edition by Leo F. Boron, P. Noordhoff, Groningen (1961)

19. Kwapień, S., Woyczyński, W.A.: Random series and stochastic integrals: Single and Multiple. Probability and its Applications. Birkhäuser Boston, Boston (1992)

20. Ledoux, M., Talagrand, M.: Probability in Banach spaces, Ergebnisse der Mathematik und ihrer Grenzgebiete (3) (Results in Mathematics and Related Areas (3)), Isoperimetry and processes, vol. 23. Springer, Berlin (1991)

21. Maas, J.: Malliavin calculus and decoupling inequalities in Banach spaces. http://arxiv.org/ abs/0801.2899

22. Metafune, G., Pallara, D., Priola, E.: Spectrum of Ornstein-Uhlenbeck operators in $L^{p}$ spaces with respect to invariant measures. J. Funct. Anal. 196(1), 40-60 (2002) 
23. Nualart, D.: The Malliavin Calculus and Related Topics, 2nd edn. Probability and its Applications (New York), Springer, Berlin (2006)

24. Rao, M.M., Ren, Z.D.: Theory of Orlicz spaces, Monographs and Textbooks in Pure and Applied Mathematics, vol. 146. Marcel Dekker, New York (1991)

25. Revuz D., Yor, M.: Continuous martingales and Brownian motion, Grundlehren der Mathematischen Wissenschaften (Fundamental Principles of Mathematical Sciences), vol. 293. Springer, Berlin (1991)

26. Rosiński, J., Suchanecki, Z.: On the space of vector-valued functions integrable with respect to the white noise. Colloq. Math. 43(1), 183-201 (1980, 1981)

27. Roynette, B.: Mouvement brownien et espaces de Besov. Stoch. Stoch. Rep. 43(3-4), 221-260 (1993)

28. Triebel, H.: Interpolation Theory, Function Spaces, Differential Operators, 2nd edn. Johann Ambrosius Barth, Heidelberg (1995)

29. Zaanen, A.C.: Riesz spaces. II. North-Holland Mathematical Library, vol. 30. North-Holland, Amsterdam (1983) 